\title{
Event-related brain potentials and the efficiency of visual search for vertically and horizontally oriented stimuli
}

\author{
Bruno Kopp, Jasmin Kizilirmak, Carolin Liebscher, Julia Runge, and Karl Wessel \\ University of Technology Carolo-Wilhelmina Braunschweig, Braunschweig, Germany \\ and Braunschweig Hospital, Braunschweig, Germany
}

\begin{abstract}
Reports that visual search is more efficient for vertically than for horizontally shaded objects suggested that search is influenced by a priori knowledge about the source of light. In this study, we examined search for targets defined by the orientation of luminance gradients and measured event-related brain potentials (ERPs). In Experiment 1, we examined search for stimuli that comprised gradual luminance differences. Response times showed the expected orientation anisotropy effect. ERP amplitudes in the P1 latency range were slightly more positive in response to horizontally oriented stimuli, whereas P3 amplitudes were more positive in response to nonsingleton vertically oriented stimuli. Experiment 2 compared search for stimuli that comprised gradual versus step differences in luminance. All the anisotropies that we observed in Experiment 1 could be replicated in Experiment 2. Moreover, these anisotropies were not dependent on the type of the luminance gradient. This finding is inconsistent with the view that search efficiency is influenced by a priori knowledge about the source of light. The behavioral and electrophysiological data are consistent with a context model of visual search. We propose that contextual modulation reduces redundancy and contributes to computing the saliency of visual information by implementing divisive normalization and multiplicative filtering.
\end{abstract}

Investigating the efficiency of visual search (VS) is a popular method that is employed by many students of cognitive processes related to visual perception (for reviews, see Wolfe, 1998; Wolfe \& Horowitz, 2004). VS typically requires searching through a display for a particular object (the target item) among other objects (the distractor items). Wolfe (2007) specified a number of basic phenomena of VS. Among the most important VS phenomena are (1) search response times (RTs) are often longer when the search display contains more distractor items (Palmer, 1995); (2) the average RT is typically longer when no target is present (Chun \& Wolfe, 1996); and (3) a limited set of visual features support very efficient VS (see Wolfe \& Horowitz, 2004, for a review). As a rule, VS is more efficient the lesser the similarity between target items and distractor items (Duncan \& Humphreys, 1989). Conversely, VS is less efficient the more heterogeneous the distractor items (Duncan \& Humphreys, 1989).

The direction of curvature is one of the basic features that support efficient VS (Treisman \& Gormican, 1988; Wolfe, Yee, \& Friedman-Hill, 1992). If the curves are part of the bounding contour of an object, this induces concavity or convexity, with observers possibly preferring concavities (Barenholtz, Cohen, Feldman, \& Singh, 2003). Taken into three dimensions, the concavity and convexity of surfaces might be basic features for studies that argue that shading is a discrete feature for VS (Aks \& Enns,
1992; Kleffner \& Ramachandran, 1992; Ramachandran, 1988; Ramachandran \& Rogers-Ramachandran, 2008).

Smooth shading gradients - that is, continuous changes in luminance - provide the visual system with information concerning the surface shape of objects with respect to the source of light. Shape-from-shading describes the phenomenon in which the visual system discerns the three-dimensional shape of an object from the shading on its surface (Prados \& Faugeras, 2006). For example, observers usually see three of the objects in the upper left panel of Figure 1 as concave and the lower left object as convex. In several studies, the effects of the orientation of shading gradients on the efficiency of VS have been examined (Adams, 2007; Kleffner \& Ramachandran, 1992; Sun \& Perona, 1998; Thornton \& Gilden, 2007; Wolfe, Klempen, \& Shulman, 1999). In these studies, empirical evidence of an orientation anisotropy was repeatedly found: VS is more efficient when the shading gradients of targets and distractors are vertically oriented (cf. the upper left panel of Figure 1), whereas VS becomes more difficult when shading gradients are horizontally oriented (cf. the upper right panel of Figure 1).

Standard theories of VS cannot account for the observed orientation anisotropy in these shape-from-shading VS studies. Feature integration theory, for example, was proposed to explain different RT slopes for feature and conjunction searches (Treisman \& Gelade, 1980). Ac-

B. Kopp, b.kopp@klinikum-braunschweig.de 


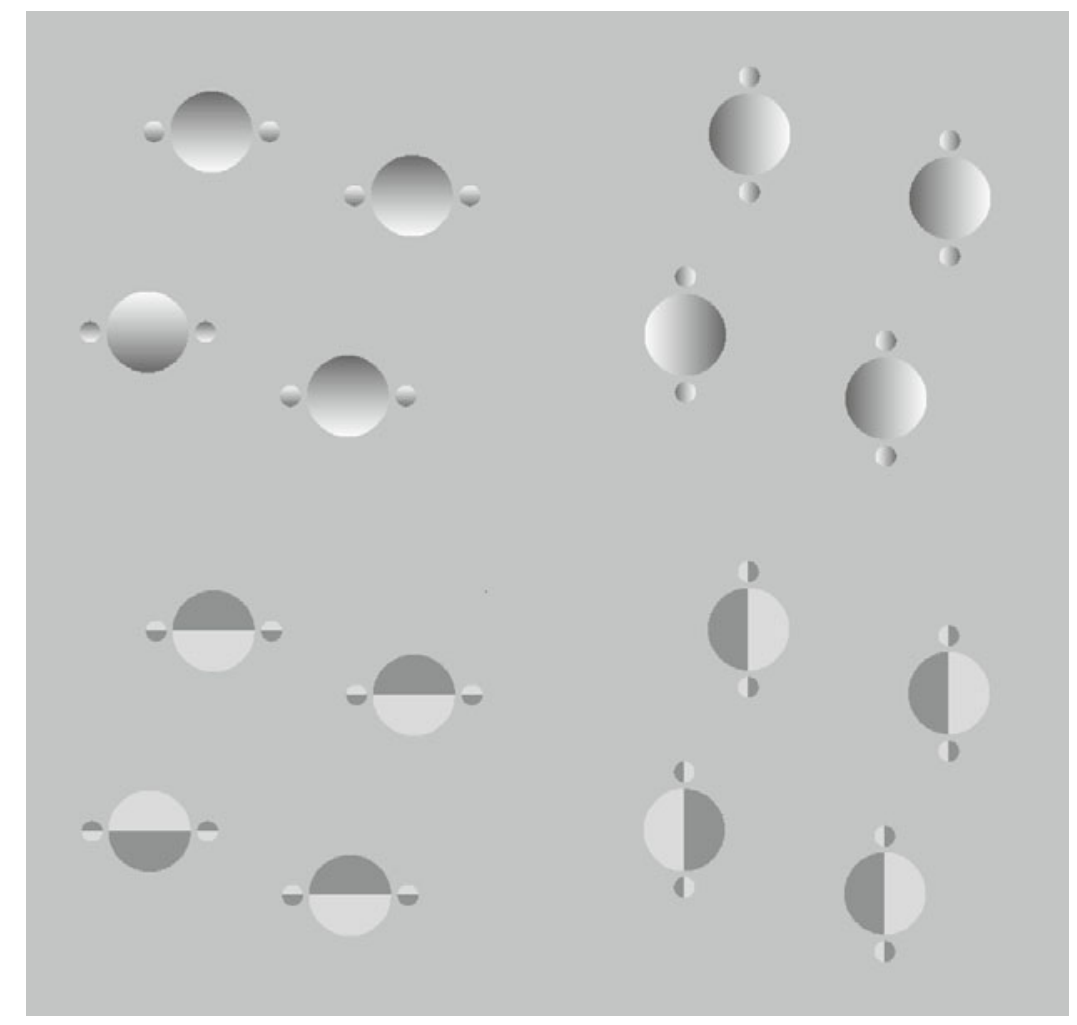

Figure 1. Four exemplary visual search displays, each with four items containing one target stimulus (i.e., the stimulus in the lower left corner). Left panels: Vertical orientation. Right panels: Horizontal orientation. Upper panels: Shape-from-shading stimuli. Lower panels: Bipartitioned stimuli. Upper left panel: Vertical shape-from-shading condition. Upper right panel: Horizontal shape-from-shading condition. Lower left panel: Vertical bipartitioned condition. Lower right panel: Horizontal bipartitioned condition.

cording to this theory, basic visual features are processed automatically and in parallel without focal attention (i.e., preattentively). In contrast, when two or more visual features belonging to the same object have to be integrated, an additional process is required that is serial in nature and comprises focal attention. There is, however, no obvious rationale for why the perpendicular stimulus rotation discussed above should lead to two qualitatively different ways of visual processing, all other things being equal (i.e., psychophysical stimulus and task variables). In another popular VS theory, preattentive processes direct the deployment of attention to interesting spatial locations in the visual field (Wolfe, 2007). According to this guided search model, stimulus-driven (bottom-up) and taskdriven (top-down) stimulus processing interact to create an activation map of attentional priority. VS is more efficient the higher the attentional priority of the target item, as compared with the attentional priorities of the distractor items. However, there is again no obvious rationale for why a sole perpendicular stimulus rotation should change the attentional priorities of targets and distractors.

Students of orientation anisotropy in these shape-fromshading VS studies sought to explain the empirically derived horizontal efficiency disadvantage by assuming that a priori knowledge influences visual perception. Specifi- cally, one of the best-known examples of a prior belief in visual perception is the assumption that light is coming from above (Kersten, Mamassian, \& Yuille, 2004). This light-from-above prior is used to recover shape from otherwise ambiguous shading (Brewster, 1826; Ramachandran, 1988; Ramachandran \& Rogers-Ramachandran, 2008). According to the light-from-above account, the orientation anisotropy arises because the assumption of overhead lighting aids one to perceive 3-D shape more vividly in the case of vertical than in the case of horizontal shading gradients (Adams, Graf, \& Ernst, 2004). Thus, implementing a priori knowledge provides visual perception with additional cues (i.e., concavity, convexity) when shading gradients are vertically oriented, in contrast to when they are horizontally oriented.

We performed two experiments. In the first one of these, event-related brain potentials (ERPs) were measured in a study in which shape-from-shading stimuli were presented and in which orientation anisotropy could be replicated successfully. The second experiment was specifically designed to evaluate a theoretical alternative to the light-from-above account for orientation anisotropy. Rather than conceptualizing anisotropy as reflecting the incorporation of prior knowledge into visual perception, the newly developed context model of anisotropy is based 
on general principles of lateral inhibition in visual processing (see the Discussion section in Experiment 2 and also Z. Li, 1999, 2002, and Nothdurft, 1991, for related models of VS). The logic behind the test of these two competing accounts was that the light-from-above account predicts that anisotropy occurs only if shape-from-shading stimuli are presented in this kind of VS studies. In sharp contrast, the context model predicts that the anisotropy that has repeatedly been demonstrated with shape-from-shading stimuli should also appear, with similar strength, with all sorts of other vertically and horizontally oriented stimuli.

The first experiment employed a VS paradigm in which circular disks with shading gradients running either from top to bottom (vertical-shading gradient) or from left to right (horizontal-shading gradient) served as stimuli (Thornton \& Gilden, 2007). These stimuli were shaded from white to black. In the vertical-shading task, they were perceived as surface bumps (white above stimulus) or as surface dimples (white below stimulus). The horizontal-shading task was based on the same stimulus set as that used in the vertical-shading task, except that the target and distractor elements were rotated $90^{\circ}$ counterclockwise. The RT data in an earlier study in which these stimuli were used (Thornton \& Gilden, 2007) showed that VS was more efficient in the vertical-shading task than in the horizontal-shading task - that is, a behavioral orientation anisotropy effect. In line with the light-from-above account, Thornton and Gilden argued that while a bias for overhead lighting might induce shape-from-shading (experience of concavity or convexity, respectively) in the vertical-shading task, the light-from-above assumption might not influence perceptual experience in the horizontal-shading task, thereby removing distinctions in surface curvature and reducing the task to a strict judgment of shading polarity.

We adopted Thornton and Gilden's (2007) vertical- and horizontal-shading tasks in our study. We assessed ERPs in addition to behavioral performance measures, in an effort to examine the cortical mechanisms of visual processing in these shape-from-shading VS tasks. The ERP technique allows one to measure cortical activity with excellent temporal resolution (Luck, 2005). The P3 (or P300) is perhaps the most-studied ERP component (for reviews, see Kok, 2001; Kopp, 2008). It is generally accepted that a distinction can be made between two subcomponentsnamely, the novelty P3 (P3a) and the target P3 (P3b, or "classical" P3; Spencer, Dien, \& Donchin, 2001). The P3b (the component focused on in the present study, further referred to as P3) has a posterior-parietal scalp distribution. There is, as of yet, no consensus about what cognitive or cortical process the P3 reflects (Luck, 2005). The hallmark of the P3 amplitude is that it is sensitive to target probability: The P3 amplitude gets larger as the target probability gets smaller (Kopp, 2008). Moreover, it has been repeatedly shown that it is the probability of the task-defined stimulus class that matters, not the probability of the physical stimulus (Kopp, 2008). Therefore, the P3 component of the ERP must be generated after the stimulus has been categorized, according to the rules of the task (Luck, 2005). Kok, in his extensive review of P3 studies, reported, as the main finding emerging from P3 studies in VS tasks, that display size affects P3 amplitude (the more items in a display, the smaller the P3 amplitude; but see Luck \& Hillyard, 1990, for divergent findings).

We also recorded visual evoked potentials (VEPs; see Hillyard, Teder-Sälejärvi, \& Münte, 1998, for a review). The first VEP component that we measured was a positivity termed P1. The P1 is usually measured at parietooccipital electrodes, and it reflects activity of extrastriate areas in the 85- to 130-msec range (Di Russo, Aprile, Spitoni, \& Spinelli, 2007; Di Russo, Martínez, Sereno, Pitzalis, \& Hillyard, 2002). The second VEP component that we measured was the posterior N1 (140-200 msec at parieto-occipital electrodes) component (cf. Di Russo et al., 2007).

Here, we conducted two VS experiments in order to challenge the replicability (Experiment 1 ) and the specificity (Experiment 2) of behavioral orientation anisotropy. Furthermore, both of these experiments were designed to investigate whether or not behavioral anisotropy is accompanied by cortical orientation anisotropy at various levels of processing by measuring VEPs and the P3 component of the ERP in shape-from-shading VS studies for the first time ever.

\section{EXPERIMENT 1}

\section{Method}

\section{Participants}

Sixteen healthy undergraduate students at the University of Technology Braunschweig participated in the experiment (two male; age, $20-44$ years; $M=24.4$ years). They volunteered to participate in return for course credit. Fifteen of them were right-handed. All the participants had normal or corrected-to-normal visual acuity. They were naive with regard to the issues in the study.

\section{Stimuli, Display, and Apparatus}

We used stimulus materials identical to those in Thornton and Gilden (2007). Circular disks, illustrated in the upper panels of Figure 1 , subtended $1.5^{\circ}$ of visual angle. Some of these shaded disks convey an impression of depth based exclusively on subtle variations (gradients) in luminance. Small reverse-shaded inducer elements were included so as to increase the percept of shape - in particular, in singleton (set size $=1$ ) displays. The orientation of the gradient was either vertical or horizontal. The shading gradient either ran from top to bottom (white to black), perceived as surface bumps, or had the opposite polarity (white to black from bottom to top), perceived as surface dimples, when the orientation of the gradient was vertical. The shading gradient either ran from left to right (white to black) or had opposite polarity (white to black from right to left), when the orientation of the gradient was horizontal. This orientation of the gradient is thought to reduce distinctions in surface curvature and converts the task mainly to a judgment of shading polarity.

The shaded disks were configured about a central fixation point along a virtual circle with a radius that subtended $2^{\circ}$ of visual angle. Disks were drawn at canonical locations along the virtual circle $\left(45^{\circ}\right.$, $\left.-45^{\circ}, 135^{\circ},-135^{\circ}\right)$. Items were distributed randomly to one of these four positions in cases in which fewer than four items were presented within a display. The entire display was rotated about fixation to remove configural effects by choosing a uniform deviate from the interval $\pm 25^{\circ}$. Disks were presented against a gray background (RGB color code: $178,178,178)$. The contrast relation between disks and background was such that some regions of the disks were brighter than the background and others were darker than the background (Aks \& Enns, 1992). 
Table 1

Number of Trials, Separately for Set Size and Number of Targets

\begin{tabular}{ccrccc}
\hline \multirow{2}{*}{ Set } & \multicolumn{5}{c}{ Number of Targets } \\
\cline { 2 - 5 } Size & \multicolumn{1}{c}{1} & \multicolumn{1}{c}{1} & 4 & Total \\
\hline 1 & 144 & 144 & - & - & 288 \\
2 & 144 & 72 & 72 & - & 288 \\
4 & 144 & 48 & 48 & 48 & 288 \\
Total & 432 & 264 & 120 & 48 & 864 \\
\hline
\end{tabular}

There were nine basic types of stimulus displays. Each display contained either one, two, or four disks (set size; displays containing three disks were excluded from this design). Displays consisted exclusively of distractors (target-absent trials), of targets (pure target-present trials), or of some combination of a variable number of targets and distractors (mixed target-present trials). Table 1 shows the frequencies of encountering each type of stimulus display. This particular design matrix was necessary to ensure that the probability of target-present and target-absent displays was balanced across the three set size conditions.

The stimuli were displayed on a 19-in. CRT monitor with a high refresh rate $(100 \mathrm{~Hz}$ at a resolution of $1,280 \times 1,024$ pixels $)$. The experimental protocol was carried out by a Presentation (Neurobehavioral Systems, Albany, CA) program written on a personal computer (PC). Manual responses were executed on both Ctrl buttons of a wireless keyboard (Logitech, Romanel-sur-Morges, Switzerland) and were recorded by the Presentation program. The left Ctrl key was pressed by the index finger of the left hand, whereas the index finger of the right hand was used to press the right Ctrl button. Stimulus displays were preceded by a fixation stimulus (250-msec duration of presentation; 1,000-msec stimulus onset asynchrony). The stimulus displays remained present until response. Response-stimulus intervals were fixed at $1,000 \mathrm{msec}$.

\section{Task and Procedure}

In the most common version of VS task, observers conduct speeded searches to determine whether a single target element is or is not present in a display of distractor elements. Multiple target search augments the standard design by including trials with more than one target (Table 1). The participant's task remains the same as in single-target search - that is, to indicate whether any targets are present.

The assignment of targets and distractors was counterbalanced across participants. Eight participants searched white above and white left disks for targets, whereas the remaining 8 participants searched white below and white right disks for targets. The assignment of response buttons to target and distractor decisions was likewise counterbalanced across participants.

Each participant completed four blocks of trials (432 trials each). The spatial orientation of the shading gradients was manipulated blockwise: Two of the blocks made use of vertically shaded disks, and the other two blocks made use of horizontally shaded disks. The sequence of shading orientations was counterbalanced across participants in such a way that 8 participants performed the two vertical blocks before the two horizontal blocks, whereas the remaining 8 participants started with the horizontal blocks and finished the experiment with the vertical blocks. The performance of a block of trials lasted about $15 \mathrm{~min}$. Short breaks of about $5 \mathrm{~min}$ separated the blocks. The experiment was carried out in a dimly lit room in which the participants sat $1 \mathrm{~m}$ away from the CRT. In order to familiarize the participants with the demands of the task, a few practice trials were administered for each shading orientation condition before the experiment actually began.

\section{Electrophysiology}

Continuous EEG was recorded by means of another PC, a QuickAmps-72 amplifier (Brain Products, Gilching, Germany) and the BrainVision Recorder Version 1.02 software (Brain Prod- ucts, Gilching, Germany) from frontal (F7, F3, Fz, F4, F8), central (T7, C3, Cz, C4, T8), parietal (P7, P3, Pz, P4, P8), occipital (O1, $\mathrm{O} 2$ ), and mastoid (M1, M2) sites. $\mathrm{Ag}-\mathrm{AgCl} \mathrm{EEG}$ electrodes were used. They were mounted on an EasyCap (EasyCap, HerrschingBreitbrunn, Germany). Electrode impedance was kept below $10 \mathrm{k} \Omega$ (mean impedance: $4 \mathrm{k} \Omega$ ). All EEG electrodes were referenced to average reference. Participants were informed about the problem of noncerebral artifacts, and they were encouraged to reduce them (Picton et al., 2000). Ocular artifacts were monitored by means of bipolar pairs of electrodes positioned at the sub- and supraorbital ridges (vertical electrooculogram, vEOG) and at the external ocular canthi (horizontal electrooculogram, hEOG). The EEG and EOG channels were amplified with a band-pass of $0.01-30 \mathrm{~Hz}$ and were digitized at $250 \mathrm{~Hz}$.

Offline analysis was performed by means of the BrainVision Analyzer Version 1.05 software (Brain Products, Gilching, Germany). Manual artifact rejection was performed before averaging in order to discard trials during which an eye movement or any other noncerebral artifact occurred. Semiautomatic blink detection and the application of an established method for ocular artifact removal were employed for ocular correction (Gratton, Coles, \& Donchin, 1983). A digital high-pass filter was applied to the data $(0.75 \mathrm{~Hz}, 48 \mathrm{~dB} /$ oct $)$ in order to eliminate low-frequency variations in the EEG signal that were associated with the occasional occurrence of electrodermal artifacts. The EEG was then divided into epochs of 1,000-msec duration, starting $100 \mathrm{msec}$ before the display onset. The prestimulus baseline of $100 \mathrm{msec}$ was subtracted from the sampling points. The EEG was averaged offline. Error trials (misses, false alarms) were excluded from averaging. Deflections in the averaged EOG waveforms were small, indicating that fixation was well maintained.

\section{Data Analysis}

Behavioral performance. Behavioral task performance was quantified in two ways. First, the median of the response speed for each table cell of the design matrix (Table 1) was computed for each individual participant, and these median individual RTs were subjected to statistical analysis. ${ }^{1}$ Second, the accuracy of the behavioral responses was computed for each table cell of the design matrix (Table 1) for each individual participant. Percentage of misses was computed for pure and mixed target-present trials. Percentage of false alarms was computed for target-absent trials. All these percentages were transferred into the arcsin transformation prior to statistical analysis.

ERPs. The amplitude and latency of the P1 and N1 peaks were measured at the amplitude peak in the intervals from 80 to $200 \mathrm{msec}$ (P1) and from 140 to $240 \mathrm{msec}(\mathrm{N} 1)$ at two parietal (P7, P8) and two occipital $(\mathrm{O} 1, \mathrm{O} 2)$ electrodes. Individual amplitudes and latencies were determined for each electrode. These measures were derived from stimulus-locked averages. Amplitude was measured as the difference between peak amplitude and the mean voltage during $100-\mathrm{msec}$ prestimulus baseline; latency was measured relative to stimulus onset.

Since the time elapsing between stimulus onset and the participant's final decision is highly variable in VS, the ERP components related to search-terminating decisions, such as the P3, were expected to be poorly time-locked to stimulus onset. However, much less variance was expected in the time-locking between these ERP waves and the behavioral response. Therefore, two types of $\mathrm{P} 3$ averages were calculated (Luck, 2005), one in which stimulus onset was the time-locking event (stimulus-locked averages) and one in which the behavioral response was the time-locking event (response-locked averages).

Amplitudes and latencies of the $\mathrm{P} 3$ were measured at Pz. Latencies were measured as the time between stimulus onset and the maximum positive peak occurring between 270 and $680 \mathrm{msec}$ poststimulus, and amplitudes were measured as the area under the curve in this latency range in stimulus-locked averages. An area, rather than a peak measure, was used for the P3 because the greater latency variation expected for larger set sizes and for the horizontal-shading condi- 
tion can distort peak amplitude measures (Luck, 2005). In responselocked averages, P3 latencies were measured as the time between the motor response and the maximum positive peak occurring in the window between $200 \mathrm{msec}$ preresponse and $200 \mathrm{msec}$ postresponse; P3 amplitudes were measured as the area under the curve within this latency range.

Statistical analyses. Behavioral and electrophysiological measures were analyzed with repeated measures ANOVAs, carried out at the .01 significance level and adjusted for nonsphericity with the Hyunh-Feldt epsilon coefficient. The results of the univariate tests are provided, using a format that gives the uncorrected degrees of freedom (Picton et al., 2000). A measure of effect size, $\eta_{\mathrm{p}}^{2}$ (partial eta squared), is also provided.

Behavioral measures (RTs, response accuracy) in one-targetpresent trials (see Table 1) were analyzed with two within-subjects factors: shading orientation (vertical, horizontal) and set size (one, two, or four items). The analyses of target-absent trials and pure target-present trials comprised an additional within-subjects factor - namely, target (target absent, pure target present).

P3 measures were analyzed with three within-subjects factors: shading orientation (vertical, horizontal), set size (one, two, or four items), and target (target absent, target present). The analysis of P1 and N1 measures comprised two additional within-subjects factorsnamely, location (occipital, parietal) and hemisphere (left, right).

\section{Results}

\section{Behavioral Performance}

One-target-present trials. First, we consider the results from the one-target-present trials (see Table 1). RTs and error rates are summarized in Figure 2 (left panels). RTs were shorter for targets in the vertical-shading task than in the horizontal-shading task; the difference was $5 \mathrm{msec}$ in the set size one condition, $65 \mathrm{msec}$ in the set size two condition, and $91 \mathrm{msec}$ in the set size four condition. The RT results led to a significant shading orientation $\times$ set size interaction effect $[F(2,30)=8.89, p<$ $.003, \eta_{\mathrm{p}}^{2}=.37, \varepsilon=.88$; see Figure 2, upper left panel]. Response accuracy was also higher in the vertical-shading task than in the horizontal-shading task, especially in the set size four condition, resulting in a significant shading orientation $\times$ set size interaction $[F(2,30)=10.67, p<$ $.002, \eta_{\mathrm{p}}^{2}=.42, \varepsilon=.82$; see Figure 2, lower left panel]. These data show a behavioral orientation anisotropy effect that gradually evolves as the VS displays contain more and more stimuli.

Target-absent trials and pure target-present trials. Next, we consider the results from those trials on which all the stimuli were distractors (target-absent trials) or targets (pure target-present trials), respectively (see Table 1). RTs and error rates are summarized in Figure 2 (right panels). RTs were generally shorter for pure targetpresent trials than for target-absent trials. This difference was more pronounced in the horizontal-shading task than in the vertical-shading task, particularly at the largest set size. The results led to a significant shading orientation $X$ set size $\times$ target interaction $[F(2,30)=9.85, p<.003$, $\eta_{\mathrm{p}}^{2}=.40, \varepsilon=.77$; see Figure 2, upper right panel]. Response accuracy was higher in pure target-present trials than in target-absent trials, especially in nonsingleton (set
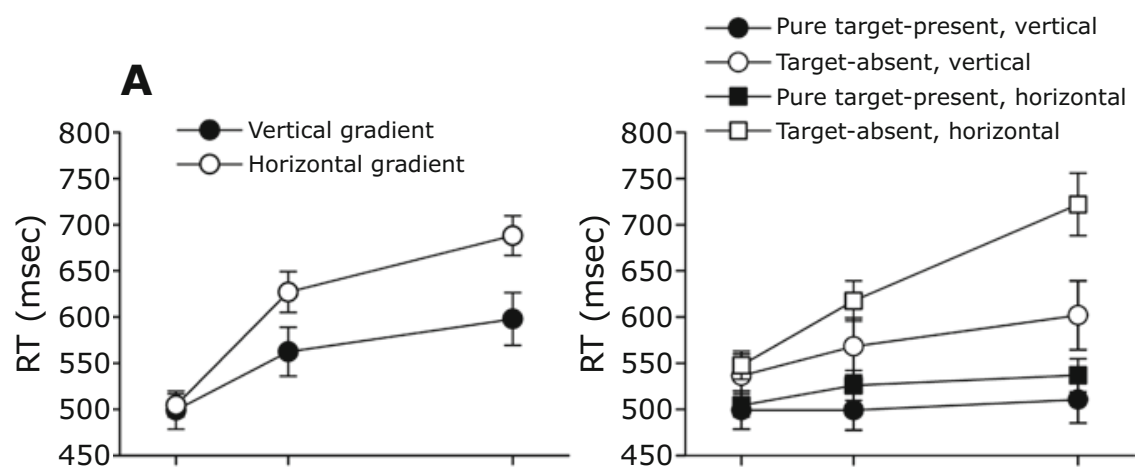

B
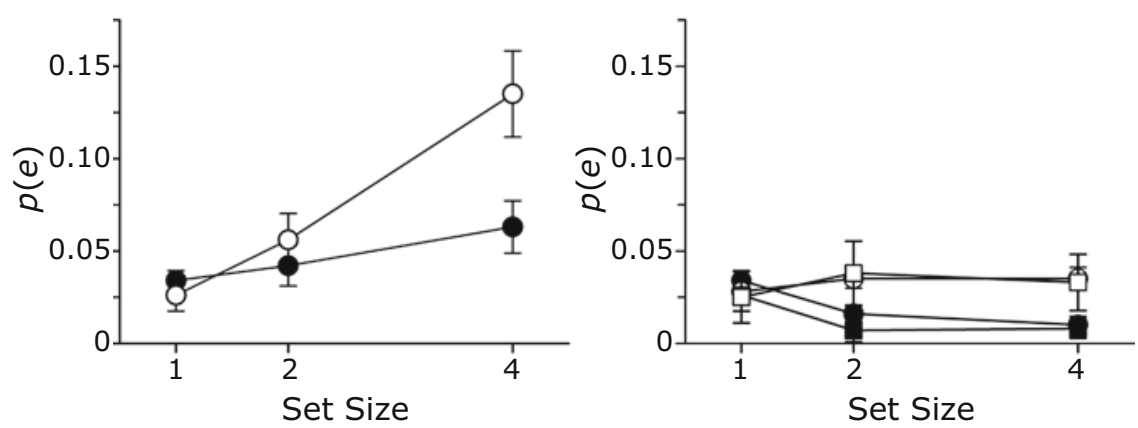

Figure 2. Behavioral results $(M \pm S E)$ obtained in Experiment 1: response times (RTs) and proportions of errors $[p(\mathrm{e}) \mathrm{s}]$. Left panels: RTs (upper panel) and $p(\mathrm{e}) \mathrm{s}$ (lower panel) from onetarget-present trials, in the vertical- and the horizontal-shading conditions and as a function of set size (one, two, and four items). Right panels: RTs (upper panel) and $p$ (e)s (lower panel) on pure target-present and target-absent trials, in the vertical- and horizontal-shading conditions and as a function of set size (one, two, and four items). 
size $>1)$ VS displays, resulting in a significant set size $\times$ target interaction $\left[F(2,30)=8.09, p<.003, \eta_{\mathrm{p}}^{2}=.35\right.$, $\varepsilon=1.0$; see Figure 2, lower left panel]. The RT data again show a behavioral orientation anisotropy effect that gradually evolves as the VS displays contain more and more stimuli and that is much more distinct on target-absent trials than it is on pure target-present trials.

\section{ERPs}

P1/N1 amplitude and latency measures. The ERPs elicited at parieto-occipital electrodes by stimulus displays of varying set size (one, four), collapsed over the two orientation conditions (vertical, horizontal), are displayed in Figure 3 (upper panels). The P1 and N1 peaks tended to decrease in amplitude and latency as set size increased, probably because of changes in stimulus parameters, rather than changes in decision processes. The set size main effect was significant for P1 latencies $[F(2,30)=63.71, p<.001$ (four shorter than one), $\left.\eta_{\mathrm{p}}^{2}=.81, \varepsilon=.69\right]$, N1 amplitudes $[F(2,30)=51.56, p<.001$ (four more negative than one), $\left.\eta_{\mathrm{p}}^{2}=.78, \varepsilon=.71\right]$, and N1 latencies $[F(2,30)=44.81, p<$ $.001, \eta_{\mathrm{p}}^{2}=.75, \varepsilon=1.0$ (four shorter than one)].

\section{Experiment 1}
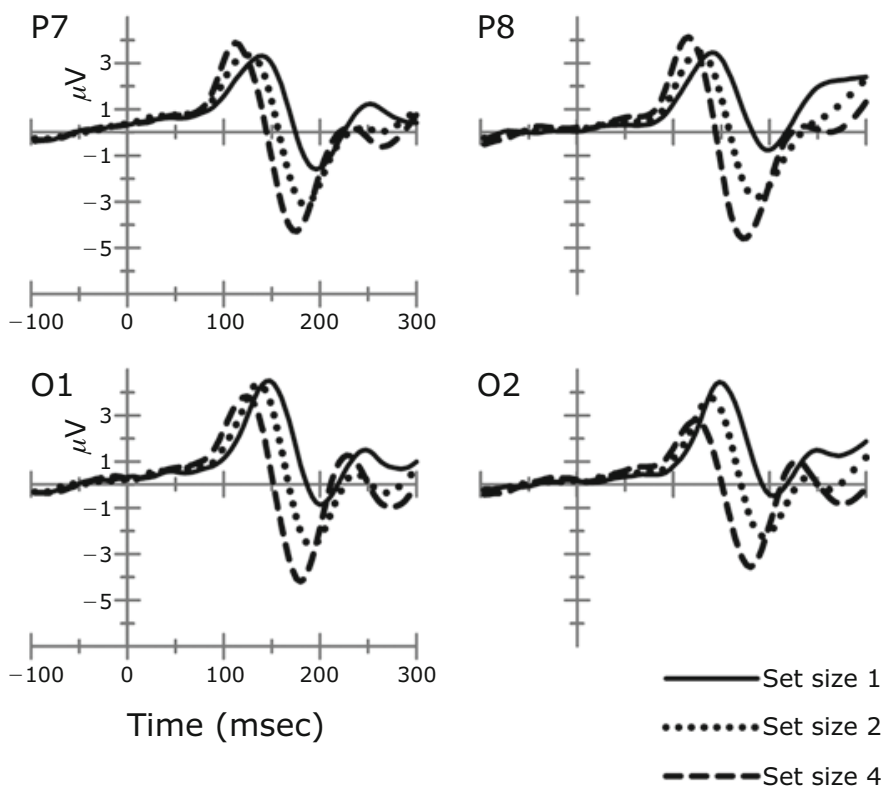

\section{Experiment 2}
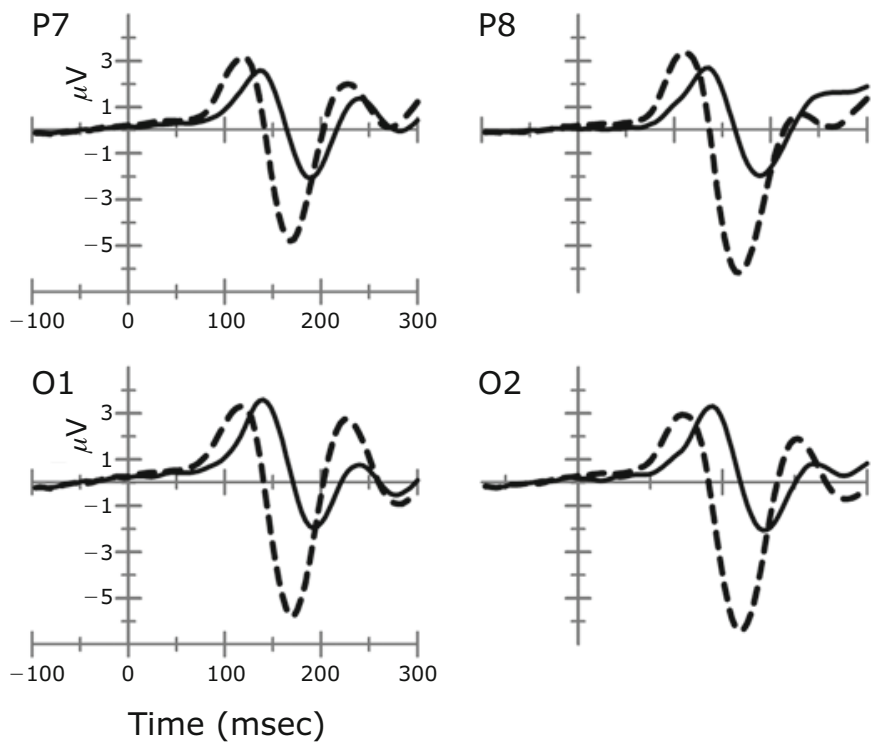

Figure 3. Grand average visual evoked potentials at parieto-occipital electrodes as a function of set size, obtained in Experiment 1 (upper panels) and in Experiment 2 (lower panels). 


\section{Experiment 1}
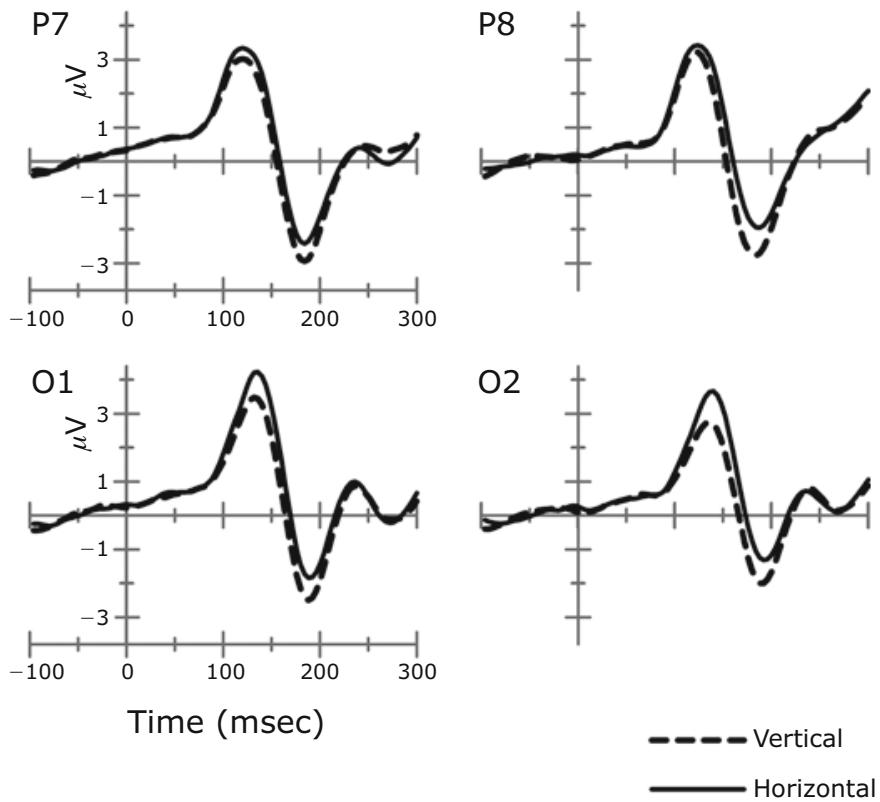

\section{Experiment 2}
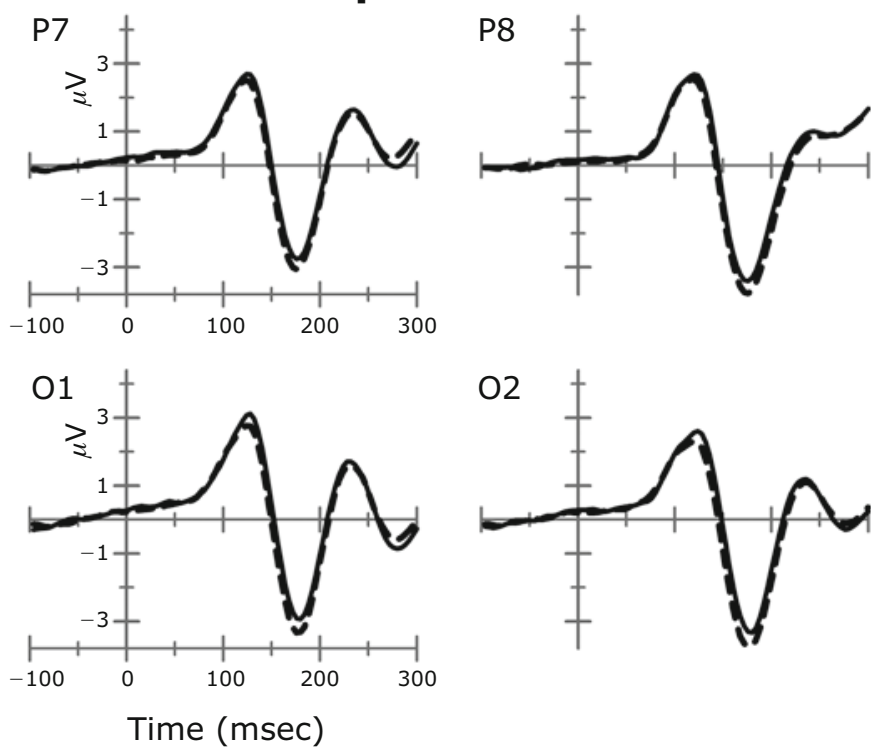

Figure 4. Grand average visual evoked potentials at parieto-occipital electrodes for vertically and horizontally oriented stimuli obtained in Experiment 1 (upper panels) and in Experiment 2 (lower panels).

P1 amplitudes were slightly more positive and N1 amplitudes were slightly less negative in the horizontalshading task than they were in the vertical-shading task (see Figure 4, upper panels). Both peak amplitude measures showed significant orientation effects [P1 amplitudes, $F(1,15)=11.04, p<.006, \eta_{\mathrm{p}}^{2}=.42 ; \mathrm{N} 1$ amplitudes, $\left.F(1,15)=16.02, p<.002, \eta_{\mathrm{p}}^{2}=.52\right]$. These $\mathrm{P} 1$ and N1 amplitude findings demonstrate that behavioral orientation anisotropy is accompanied by cortical orientation anisotropy. Specifically, the earliest sign of cortical orientation anisotropy was observed in P1 amplitudes (horizontal P1 more positive than vertical P1). ${ }^{2}$

P3 amplitude and latency measures. Stimulus displays also elicited a large $\mathrm{P} 3$ wave with parietal maximum (see Figures 5 and 6, upper panels). Stimulus-locked averages (see Figure 5, upper left panel) showed that the target-present stimulus displays elicited more positive P3 mean amplitudes than did the target-absent stimulus displays $\left[F(1,15)=60.53, p<.001, \eta_{\mathrm{p}}^{2}=.80\right]$. P3 mean amplitudes in the response-locked averages were also enhanced in target-present trials, as compared with target- 


\section{Experiment 1}

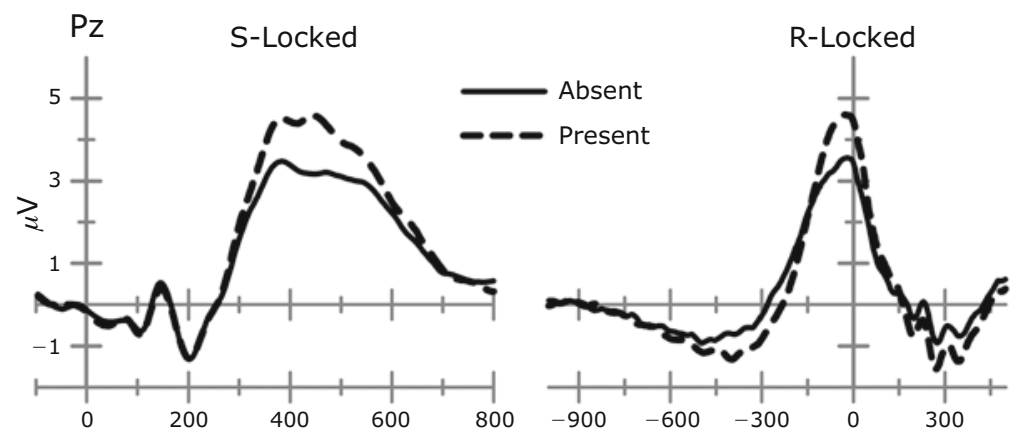

Experiment 2
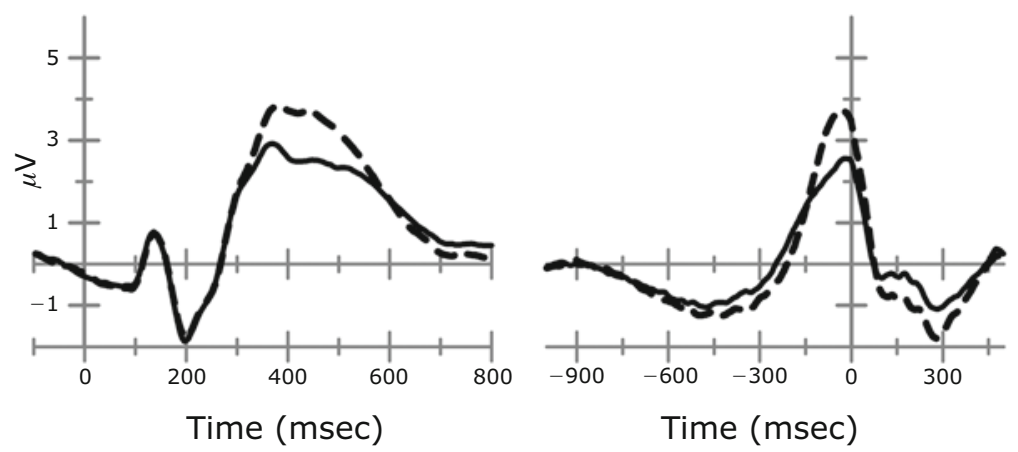

Figure 5. Grand average stimulus-locked (left panels) and response-locked (right panels) event-related brain potentials at electrode $\mathrm{Pz}$ for target-absent and targetpresent displays obtained in Experiment 1 (upper panels) and in Experiment 2 (lower panels).

absent trials $\left[F(1,15)=21.9, p<.001, \eta_{\mathrm{p}}^{2}=.59\right.$; see Figure 5, upper right panel].

Stimulus-locked P3 mean amplitudes decreased as set size increased $\left[F(2,30)=14.16, p<.002, \eta_{\mathrm{p}}^{2}=.49, \varepsilon=\right.$ .68]. Importantly, the set size effect on $\mathrm{P} 3$ amplitudes was orientation specific, because the amplitude decrement with increasing set size was much stronger in the horizontalshading task than it was in the vertical-shading task $\left[F(2,30)=13.09, p<.002, \eta_{\mathrm{p}}^{2}=.47, \varepsilon=.61\right.$; see Figure 6 , upper left panel]. This finding constitutes evidence for another cortical orientation anisotropy effect that is expressed in stimulus-locked P3 mean amplitudes (vertical P3 > horizontal P3) and that is specifically induced by nonsingleton VS displays. Response-locked P3 mean amplitudes decreased as set size increased $[F(2,30)=7.21, p<.005$, $\eta_{\mathrm{p}}^{2}=.33, \varepsilon=.88$; see Figure 6, upper right panel], without a statistically reliable modulation by shading orientation $\left[F(2,30)=1.38, p=.27, \eta_{\mathrm{p}}^{2}=.08, \varepsilon=.81\right]$.

Set size strongly affected stimulus-locked P3 peak latencies, with a mean latency of $431 \mathrm{msec}$ in the set size one condition, $459 \mathrm{msec}$ in the set size two condition, and $484 \mathrm{msec}$ in the set size four condition. This observation was corroborated by a significant set size main effect $\left[F(2,30)=12.23, p<.002, \eta_{\mathrm{p}}^{2}=.45, \varepsilon=.71\right] . \mathrm{P} 3$ peak latencies in the response-locked averages were somewhat less sensitive to the manipulation of set size: $-39 \mathrm{msec}$ (set size one), $-23 \mathrm{msec}$ (set size two), and $-28 \mathrm{msec}$ (set size four), a difference that failed to reach statistical significance $\left[F(2,30)=5.27, p=.02, \eta_{\mathrm{p}}^{2}=.26, \varepsilon=.82\right]$.

\section{Discussion}

Our behavioral results replicate the behavioral orientation anisotropy that has been reported in earlier shape-fromshading VS studies (Adams, 2007; Kleffner \& Ramachandran, 1992; Sun \& Perona, 1998; Thornton \& Gilden, 2007; Wolfe et al., 1999). Specifically, RTs and error measures revealed that VS for vertically shaded stimuli was more efficient than VS for horizontally shaded stimuli under nonsingleton display conditions. We found orientation $\times$ set size interaction RT effects in one-target-present trials, and this two-way interaction was also much stronger in targetabsent trials than it was in pure target-present trials.

This is the first study that we are aware of to report ERP measures from a shape-from-shading VS experiment. We basically found two cortical correlates of behavioral anisotropy. First, VEP amplitudes showed evidence of orientation anisotropy. Specifically, peak amplitudes in the P1 latency range were slightly, but reliably, more positive in the horizontal-shading condition than in the verticalshading condition. It is not possible at the moment to determine whether the $\mathrm{P} 1$ or the $\mathrm{N} 1$ component of the ERP, or both, or another ERP component has changed. Yet the 


\section{Experiment 1}
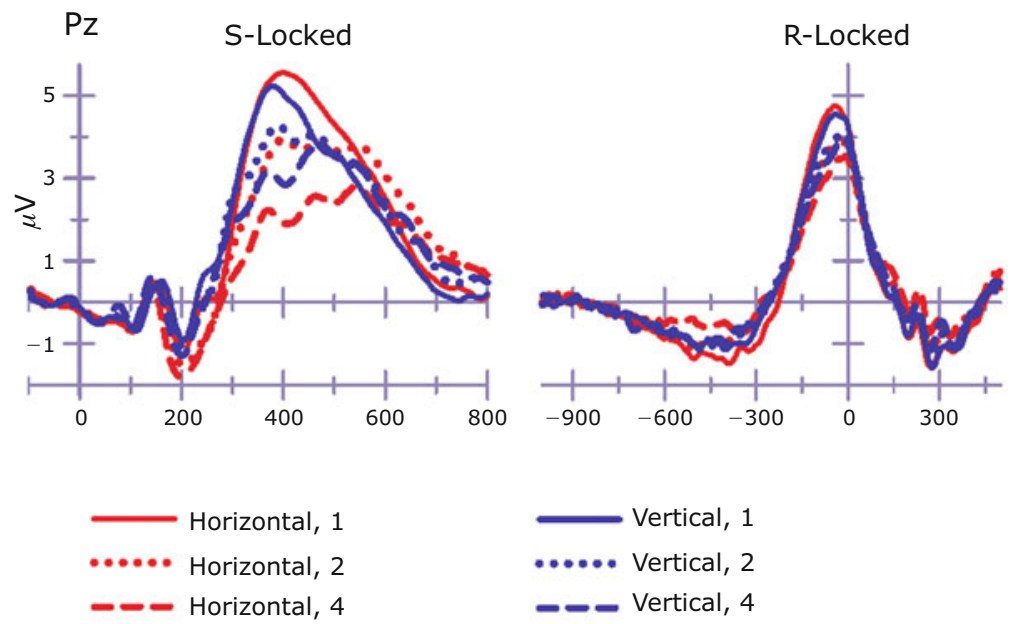

Experiment 2
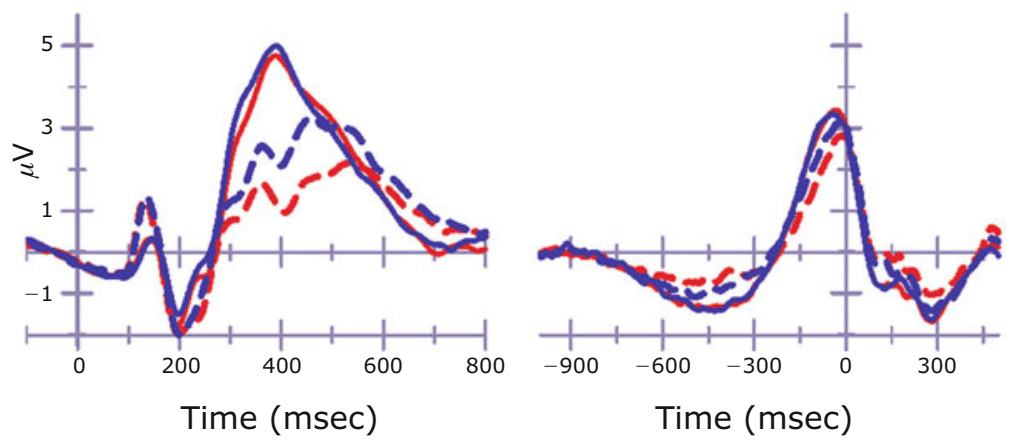

Figure 6. Grand average stimulus-locked (left panel) and response-locked (right panel) event-related brain potentials at electrode $\mathrm{Pz}$ in the vertical- (blue lines) and horizontal- (red lines) shading conditions and as a function of set size (one, two, and four items in Experiment 1 [upper panels]; one and four items in Experiment 2 [lower panels]).

change in voltage in the $\mathrm{P} 1$ latency range reflects an orientation effect over the occipital cortex in the latency range between 80 and $200 \mathrm{msec}$. Second, mean amplitudes of the P3 component of the ERP showed evidence of cortical anisotropy, since P3 amplitudes, but not latencies, were differentially affected by set size in the vertical- and the horizontal-shading conditions. Specifically, P3 amplitude decrement that is usually associated with an increase in the number of items in VS displays (Kok, 2001) was more pronounced under horizontal-shading conditions than it was under vertical-shading conditions.

A notoriously difficult issue in ERP research is whether or to what degree apparent condition or group differences in average amplitudes are due to differences in trial-totrial latency variability. With regard to this question, it is important to note that we used area-based P3 amplitude measures that are useful for mitigating the effects of trial-to-trial latency variability under most conditions, particularly when the waveforms are not multiphasic and when overlapping components do not preclude the use of a wide measurement window (Luck, 2005). The modulation of the P3 amplitude set size effect by the orientation of the shading gradient could not be observed in response-locked averages. The reasons for this effect being absent in response-locked averages are not entirely clear. According to one view, the processing difference that is reflected in the shading orientation $\times$ set size interaction in the stimulus-locked averages is time-locked to stimulus-oriented processes, rather than to responserelated processes.

Taken together, we found evidence of cortical anisotropy at multiple levels of the cortical hierarchy, and these anisotropies pointed in opposite directions. Specifically, whereas amplitudes in the P1 latency range were slightly more positive under horizontal-shading conditions, P3 amplitudes were more positive under nonsingleton, verticalshading conditions. To our knowledge, no earlier study has examined ERP measures from comparable shape-fromshading VS studies. There are only two ERP studies that have reported relevant data. Mamassian, Jentzsch, Bacon, and Schweinberger (2003) presented ambiguously shaded patterns and examined VEPs. They found correlations be- 
tween P1 amplitudes and observer biases for particular spatial positions of the assumed light source. These data were interpreted as ERP evidence that a priori knowledge about the source of light is represented early in the visual system. Hou, Pettet, Vildavski, and Norcia (2006) updated visual stimuli, thereby alternating their perceptual interpretations systematically. When stimuli were updated in asymmetric conditions, the perceptual interpretation changed from two-dimensional to three-dimensional. Symmetric updating conditions were characterized by two corrugated surfaces that looked laterally translated, without any change in depth interpretation. VEPs in response to asymmetric shifts were characterized by enhanced, sustained negative waveforms in the latency range between 150 and $300 \mathrm{msec}$ poststimulus over the posterior scalp, as compared with VEPs in response to symmetric shifts, possibly reflecting a cortical correlate of depth perception. Taken together, these studies showed that a priori knowledge about the source of light and depth perception modulate VEP amplitudes in the $\mathrm{P} 1$ and N1 latency range.

There is only one published study that examined neurophysiological correlates of shape-from-shading in VS. Lee, Yang, Romero, and Mumford (2002) recorded spiking responses of $\mathrm{V} 1$ and $\mathrm{V} 2$ neurons of macaque monkeys. Their task was to fixate a target during VS for shapefrom-shading stimuli that were similar to those used by Ramachandran (1988) and by us. V2, but not V1, neurons responded highly sensitively to the shape-from-shading stimuli, possibly indicating that V2 may be the first cortical area that is sensitive to 3-D surface shape. These neurophysiological data are in general accord with our P1 data, if one considers the extrastriate sources of the P1 component (Di Russo et al., 2007; Di Russo et al., 2002).

\section{EXPERIMENT 2}

The results of Experiment 1 showed that behavioral anisotropy is a replicable phenomenon in shape-fromshading VS studies. Specifically, VS under nonsingleton conditions is more efficient for vertically shaded stimuli than it is for horizontally shaded stimuli. Furthermore, this behavioral anisotropy may result from anisotropic cortical processing of vertical and horizontal visual stimuli, respectively, because amplitudes in the P1 latency range were more positive under horizontal shading conditions, whereas P3 amplitudes were more positive under nonsingleton vertical shading conditions.

We outlined in the introduction to this article that behavioral anisotropy is usually interpreted as resulting from perceptual pop-out effects that are putatively supported by the light-from-above assumption (Kersten et al., 2004; Ramachandran, 1988; Ramachandran \& RogersRamachandran, 2008). According to this model, the bias for overhead lighting enables the perception of vertically, but not horizontally, shaded stimuli three-dimensionally, and the availability of 3-D cues (i.e., concavity, convexity) aids efficient VS (Adams, 2007; Adams et al., 2004; Kleffner \& Ramachandran, 1992; Sun \& Perona, 1998; Thornton \& Gilden, 2007; Wolfe et al., 1999). However, the validity of the light-from-above account for the re- ported behavioral anisotropy needs to be assessed further. One caveat to this model is that it does not provide a comprehensive rationale toward understanding the observed ERP amplitude anisotropies.

An important caveat to the light-from-above account for the reported behavioral anisotropy has been provided by van Zoest, Giesbrecht, Enns, and Kingstone (2006). The authors of this study demonstrated orientation anisotropy in the absence of shape-from-shading stimuli. In three of their experiments, anisotropy was observed on vertical and horizontal displays in which the stimuli were clearly not interpretable as 3-D surfaces. The sole exception to that rule occurred in their Experiment 4, in which the elimination of interitem symmetry resulted in equal search efficiency for vertical and horizontal displays. These findings were interpreted in terms of item similarity. Specifically, it was conjectured that targets and distractors are perceived as being more similar on horizontal (as compared with vertical) displays - thereby decreasing search efficiency (Duncan \& Humphreys, 1989) and breaking ground for orientation anisotropy, except under conditions in which interitem symmetry is eliminated.

On the basis of these earlier findings, Experiment 2 served to examine whether or not behavioral and/or cortical orientation anisotropy occurs specifically in response to shape-from-shading stimuli. In order to examine the specificity of these phenomena, we employed two different types of stimuli: the smoothly and continuously graded shape-from-shading stimuli of Experiment 1 and newly constructed, discontinuous stimuli (see the lower panels of Figure 1). These bipartitioned disks contain a step difference in luminance in the vertical (cf. lower left panel of Figure 1) or in the horizontal (cf. upper right panel of Figure 1) direction. They were originally introduced by Kleffner and Ramachandran (1992) as stimuli in control VS displays that do not convey depth information, as confirmed by Aks and Enns (1992). Nevertheless, it was never examined before whether orientation anisotropy occurs when tested with this kind of bipartitioned stimuli. This was the purpose of Experiment 2.

According to the light-from-above account for orientation anisotropy in VS tasks, the 3-D interpretation of the visual stimuli presupposes vertical-shading gradients such that the bias for overhead lighting is able to support perception of concavity or convexity, respectively. In contrast, the bias for overhead lighting should be able to support the 3-D perception of stimuli neither in cases of horizontal-shading gradients - thereby giving rise to orientation anisotropy - nor in cases in which stimuli do not convey depth information (as in the case of vertical and horizontal bipartitioned stimuli). Thus, the lightfrom-above model predicts the occurrence of orientation anisotropy solely when tested with shape-from-shading stimuli, but not when tested with bipartitioned stimuli.

\section{Method}

\section{Participants}

Twenty-four healthy undergraduate students at the University of Technology Braunschweig who had not participated in Experi- 
Table 2

Number of Trials, Separately for Set Size, Target Presence Versus Absence, and Stimulus Conditions (Type of Gradient, Orientation, Polarity)

\begin{tabular}{|c|c|c|c|c|c|c|c|c|c|}
\hline & \multicolumn{8}{|c|}{ Stimulus Condition } & \multirow[b]{4}{*}{ Total } \\
\hline & \multicolumn{4}{|c|}{ Shape-From-Shading (3-D) } & \multicolumn{4}{|c|}{ Bipartitioned (2-D) } & \\
\hline & \multicolumn{2}{|c|}{ Vertical } & \multicolumn{2}{|c|}{ Horizontal } & \multicolumn{2}{|c|}{ Vertical } & \multicolumn{2}{|c|}{ Horizontal } & \\
\hline & Above & Below & Left & Right & Above & Below & Left & Right & \\
\hline \multicolumn{10}{|l|}{ Set Size 1} \\
\hline Present & 52 & 52 & 52 & 52 & 52 & 52 & 52 & 52 & 416 \\
\hline Absent & 52 & 52 & 52 & 52 & 52 & 52 & 52 & 52 & 416 \\
\hline \multicolumn{10}{|l|}{ Set Size 4} \\
\hline Present & 52 & 52 & 52 & 52 & 52 & 52 & 52 & 52 & 416 \\
\hline Absent & 52 & 52 & 52 & 52 & 52 & 52 & 52 & 52 & 416 \\
\hline Total & 208 & 208 & 208 & 208 & 208 & 208 & 208 & 208 & 1,664 \\
\hline
\end{tabular}

ment 1 volunteered in the experiment (six male; age, 20-36 years; $M=22.8$ years). Twenty-two of them were right-handed. All the participants had normal or corrected-to-normal visual acuity. They were naive with regard to the issues in the study.

\section{Stimuli, Display, and Apparatus}

The stimuli, display, and apparatus were the same as those in Experiment 1, except for the following modifications. Bipartitioned disks that comprised a step difference in luminance in the vertical (cf. lower left panel of Figure 1) or in the horizontal (cf. lower right panel of Figure 1) direction were introduced, in addition to the shape-from-shading stimuli. Shape-from-shading and bipartitioned disks possessed identical diameters. We made an attempt to hold mean luminance contrast across the two types of gradients constant by subjectively adjusting them.

\section{Task and Procedure}

The task and procedure were the same as those in Experiment 1, except for the following modifications. There were four basic types of stimulus displays. Each display contained either one or four disks (set size). Displays either consisted exclusively of distractors (target-absent trials) or contained one target (target-present trials). Thus, multiple-target trials were not applied in this experiment. Table 2 shows the frequencies of encountering each type of stimulus display. Each participant completed eight blocks of trials (208 trials each). Each block of trials comprised equal numbers of target-present and target-absent trials, as well as equal numbers of singleton and nonsingleton trials (see Table 2). The type of gradient (shape-from-shading, bipartitioned) and their spatial orientation (vertical, horizontal), as well as the polarity of the target-defining feature (white above, white below, white left, white right), were manipulated blockwise, in a counterbalanced manner. The assignment of response buttons to target and distractor decisions was likewise counterbalanced across participants. The performance of one block of trials lasted about $10 \mathrm{~min}$.

\section{Electrophysiology}

Electrophysiology was the same as in Experiment 1.

\section{Data Analysis}

Behavioral performance. Measurement of behavioral performance was the same as in Experiment 1.

ERPs. Measurement of ERPs was the same as in Experiment 1, except for the following modifications. Stimulus-locked P3 amplitudes and latencies were measured in the time window between 270 and $850 \mathrm{msec}$ poststimulus. Response-locked P3 amplitudes and latencies were measured in the time window between $200 \mathrm{msec}$ preresponse and $100 \mathrm{msec}$ postresponse.

Statistical analyses. The statistical analyses were the same as in Experiment 1, except for the following modifications. Behavioral measures (RTs, response accuracy) on target-present and targetabsent trials (see Table 2) were analyzed with five within-subjects factors: type of gradient (shape-from-shading, bipartitioned), spatial orientation (vertical, horizontal), polarity of the target-defining feature (white above, white below, white left, white right), set size (one or four items), and target (presence, absence). P3 measures were analyzed with four within-subjects factors: type of gradient (shape-from-shading, bipartitioned), spatial orientation (vertical, horizontal), set size (one or four items), and target (presence, absence). The analysis of P1 and N1 measures comprised an additional within-subjects factor-namely, location (occipital, parietal) and hemisphere (left, right).

\section{Results}

\section{Behavioral Performance}

RTs were shorter in the set size one condition than they were in the set size four condition $[F(1,23)=114.92, p<$ $\left..001, \eta_{\mathrm{p}}^{2}=.83\right]$. RTs were also shorter for target-present trials than they were for target-absent trials $[F(1,23)=$ 64.32, $\left.p<.001, \eta_{\mathrm{p}}^{2}=.74\right]$. This target effect on RTs was more pronounced for $3-\mathrm{D}$ stimuli than it was for 2 -D stimuli $\left[F(1,23)=9.66, p<.006, \eta_{\mathrm{p}}^{2}=.30\right]$. The target effect on RTs was also modulated by the interaction of orientation condition (vertical, horizontal) and set size (one, four) $\left[F(1,23)=13.38, p<.002, \eta_{\mathrm{p}}^{2}=.37\right]$. Separate ANOVAs were performed within each orientation condition to parse the three-way interaction. A significant two-way interaction of target (present, absent) and set size (one, four) emerged for horizontal conditions $\left[F(1,23)=10.04, p<.005, \eta_{\mathrm{p}}^{2}=.30\right]$. In contrast, set size and target exerted main effects only for vertical conditions $\left[F(1,23)=132.62, p<.001, \eta_{\mathrm{p}}^{2}=.85\right.$, and $F(1,23)=$ 56.81, $p<.001, \eta_{\mathrm{p}}^{2}=.71$, respectively]. Thus, the target effect on RTs showed an increment across set size conditions in the horizontal, but not in the vertical, orientation condition, leading to particularly long RTs in nonsingleton horizontal target-absent trials.

RTs were generally shorter for vertically than for horizontally oriented stimuli $[F(1,23)=35.91, p<$ $\left..001, \eta_{\mathrm{p}}^{2}=.61\right]$. As in Experiment 1, this anisotropy effect was more pronounced when nonsingleton displays (set size four), as compared with singleton displays (set size one), were presented $[F(1,23)=29.95, p<.001$, $\left.\eta_{\mathrm{p}}^{2}=.57\right]$. Importantly, set-size-dependent orientation anisotropy was clearly not modulated by type of gradient $\left[F(1,23)=1.79, p=.19, \eta_{\mathrm{p}}^{2}=.07\right]$. When separate ANOVAs were performed on each stimulus type (3-D, 2-D), a significant two-way interaction of orientation 
(vertical, horizontal) and set size (one, four) emerged for the 3-D stimuli $\left[F(1,23)=25.76, p<.001, \eta_{\mathrm{p}}^{2}=\right.$ .53], reflecting orientation anisotropy. Most important, this interaction was also significant for the 2-D stimuli $\left[F(1,23)=25.89, p<.001, \eta_{\mathrm{p}}^{2}=.53\right]$. The upper panels of Figure 7 depict the relevant mean RTs. A look at these mean RTs reveals that orientation anisotropy appeared, in similar strength, with both sorts (3-D, 2-D) of vertically and horizontally oriented stimuli. The nonspecificity of orientation anisotropy is a finding that is clearly incompatible with the light-from-above account for orientation anisotropy.

The analysis of error rates confirmed this conclusion. Error rates were influenced by set size $[F(1,23)=24.44$, $\left.p<.001, \eta_{\mathrm{p}}^{2}=.52\right]$ and by target $[F(1,23)=35.91, p<$ $\left..001, \eta_{\mathrm{p}}^{2}=.61\right]$. Type of stimuli exerted neither a main effect $\left[F(1,23)=1.10, p=.31, \eta_{\mathrm{p}}^{2}=.05\right]$, nor any significant interaction effect [all $F_{\mathrm{s}}(1,23) \leq 5.83, p \mathrm{~s} \geq .02$, $\left.\eta_{\mathrm{p}}^{2} \mathrm{~s} \leq .20\right]$, on error rates. Specifically, the three-way type of gradient $\times$ orientation $\times$ set size interaction clearly failed to reach statistical significance $[F(1,23)<1]$. In contrast, orientation (vertical, horizontal) influenced error rates $\left[F(1,23)=12.92, p<.003, \eta_{\mathrm{p}}^{2}=.36\right.$ (horizontal $>$ vertical) $]$ in interaction with the set size condition $\left[F(1,23)=37.68, p<.001, \eta_{\mathrm{p}}^{2}=.62\right.$ (four $>$ one; see Figure 7, lower panels)]. Error rates in horizontal displays of the set size four condition were particularly high when targets were absent $\left[F(1,23)=10.79, p<.004, \eta_{\mathrm{p}}^{2}=.32\right.$ (three-way interaction of orientation, target, and set size)].
This observation on error rates parallels the observation on RTs, providing convergent evidence that nonsingleton horizontal target-absent displays posed specific difficulties to the perceivers.

\section{ERPs}

P1/N1 amplitude and latency measures. The ERPs elicited at parieto-occipital electrodes by stimulus displays of varying size (one, four) are displayed in Figure 3 (lower panels). P1 and N1 peak amplitudes and latencies decreased as set size increased, probably because of changes in stimulus parameters, rather than changes in decision processes. The set size main effect reached statistical significance for P1 latencies $[F(1,23)=204.87, p<$ $\left..001, \eta_{\mathrm{p}}^{2}=.90\right], \mathrm{N} 1$ amplitudes $[F(1,23)=112.31, p<$ $\left..001, \eta_{\mathrm{p}}^{2}=.83\right]$, and N1 latencies $[F(1,23)=197.04$, $\left.p<.001, \eta_{\mathrm{p}}^{2}=.90\right]$. P1 amplitudes were affected by the two-way set size $\times$ location of electrode (occipital, parietal) interaction $\left[F(1,23)=8.90, p<.008, \eta_{\mathrm{p}}^{2}=.28\right]$.

P1 peak amplitudes were also affected by orientation $\left[F(1,23)=11.77, p<.003, \eta_{\mathrm{p}}^{2}=.34\right.$ (horizontal P1 more positive than vertical $\mathrm{P} 1$ )], thereby replicating the finding that we had obtained in Experiment 1. Importantly, the type of gradient $\times$ orientation interaction was not statistically significant $[F(1,23)<1]$. When separate ANOVAs were performed on each stimulus type (3-D, 2-D), a significant orientation effect emerged for the 2-D stimuli $[F(1,23)=$ $\left.8.02, p<.01, \eta_{\mathrm{p}}^{2}=.26\right]$, whereas the orientation effect for the 3-D stimuli merely emerged as a statistical trend
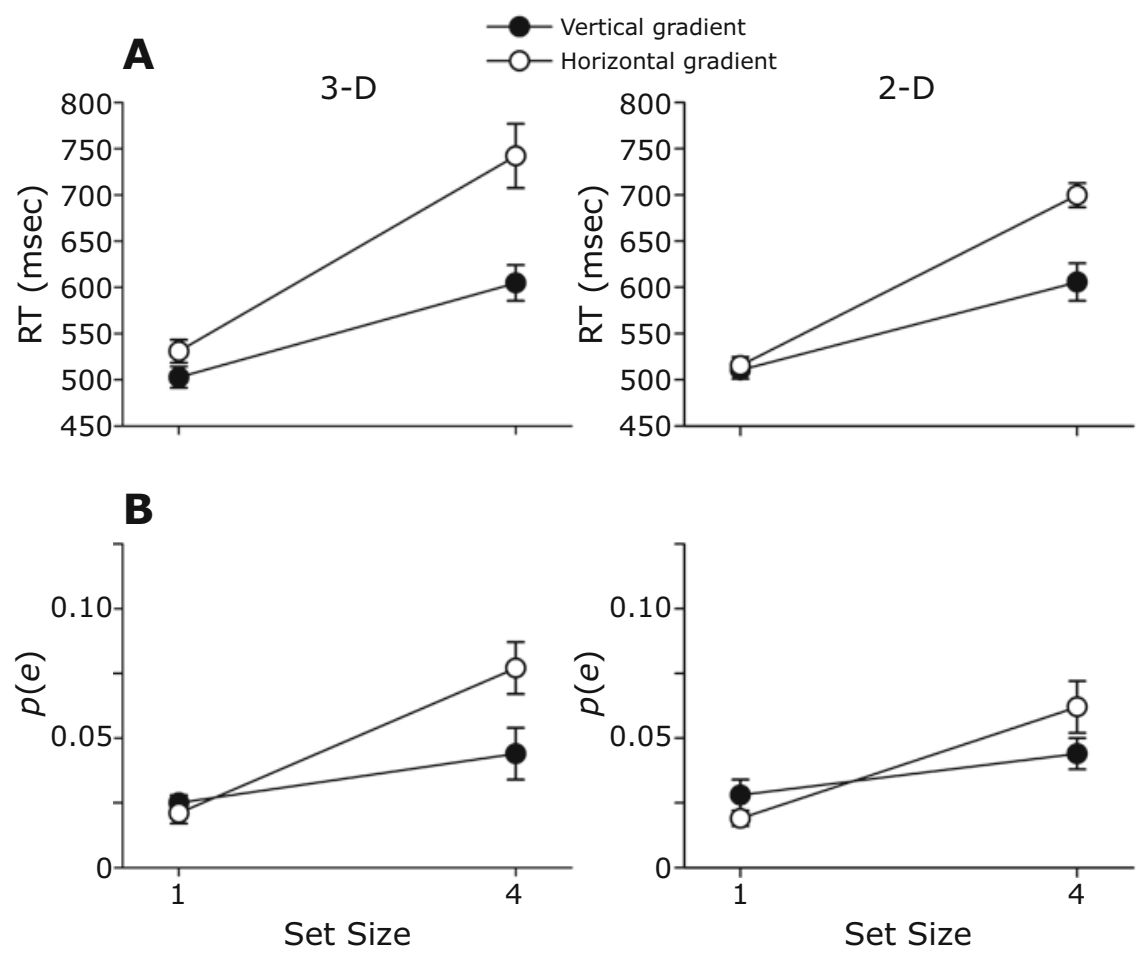

Figure 7. Behavioral results $(M \pm S E)$ obtained in Experiment 2: response times (RTs) and proportions of errors $[p(\mathrm{e}) \mathrm{s}]$. Left panels: RTs (upper panel) and $p(\mathrm{e}) \mathrm{s}$ (lower panel) for shape-from-shading (3-D) conditions. Right panels: RTs (upper panel) and $p(e) s$ (lower panel) for bipartitioned (2-D) conditions. 
$\left[F(1,23)=6.51, p=.02, \eta_{\mathrm{p}}^{2}=.22\right] . \mathrm{P} 1$ peak latencies were also affected by orientation $[F(1,23)=9.34, p<$ $.007, \eta_{\mathrm{p}}^{2}=.29$ (horizontal longer than vertical)], but again this influence of orientation was clearly not modulated by type of gradient $[F(1,23)<1]$. In separate ANOVAs on each stimulus type (3-D, 2-D), the orientation effect failed to reach statistical significance [for 2-D stimuli, $F(1,23)=$ $5.05, p=.04, \eta_{\mathrm{p}}^{2}=.18$; for 3 -D stimuli, $F(1,23)=2.64$, $\left.p=.12, \eta_{\mathrm{p}}^{2}=.10\right]$. The ANOVA on N1 amplitudes yielded a significant three-way orientation $\times$ target $\times$ set size interaction $\left[F(1,23)=23.16, p<.001, \eta_{\mathrm{p}}^{2}=.50\right]$. Type of gradient or interactions between this factor and any other factor did not reach statistical significance [all $F_{\mathrm{s}}(1,23) \leq$ $\left.6.84, p \mathrm{~s} \geq .02, \eta_{\mathrm{p}}^{2} \mathrm{~s} \leq .23\right]$. Finally, N1 latencies were unaffected by orientation, type of gradient, their interaction, and interactions between these factors and any other factors [all $F_{\mathrm{s}}(1,23) \leq 6.42, p \mathrm{~s} \geq .02, \eta_{\mathrm{p}}^{2} \leq .22$ ].

$P 3$ amplitude and latency measures. The stimulus displays also elicited large P3 waves with parietal maximum (see Figures 5 and 6, lower panels). Stimulus-locked averages showed that target-present displays elicited more positive $\mathrm{P} 3$ mean amplitudes than did target-absent displays $\left[F(1,23)=22.65, p<.001, \eta_{\mathrm{p}}^{2}=.50\right.$; see Figure 5 , lower left panel]. Response-locked P3 mean amplitudes also showed a target effect $[F(1,23)=27.22, p<.001$, $\left.\eta_{\mathrm{p}}^{2}=.54\right]$, modulated by type of stimuli (3-D, 2-D) $\left[F(1,23)=14.75, p<.002, \eta_{\mathrm{p}}^{2}=.39\right.$; see Figure 5, lower right panel].

Stimulus-locked P3 mean amplitudes decreased as set size increased $\left[F(1,23)=20.32, p<.001, \eta_{\mathrm{p}}^{2}=.47\right.$; see Figure 6, lower left panel]. Importantly, the set size effect on P3 mean amplitudes was orientation specific, because the amplitudes decreased much more with increasing set size in horizontal than in vertical conditions $[F(1,23)=$ $\left.17.44, p<.001, \eta_{\mathrm{p}}^{2}=.43\right]$. The orientation $\times$ set size interaction effect on P3 mean amplitude was independent of type of stimuli $\left[F(1,23)=3.66, p=.07, \eta_{\mathrm{p}}^{2}=.14\right]$. This finding replicates the orientation anisotropy of stimuluslocked P3 mean amplitudes (nonsingleton vertical P3 > nonsingleton horizontal P3) that we had observed in Experiment 1 . Furthermore, it shows that the orientation anisotropy of stimulus-locked P3 mean amplitudes does not occur specifically in response to shape-from-shading stimuli. This conclusion was further corroborated by two separate ANOVAs that revealed a significant two-way orientation $\times$ set size interaction for 3 -D stimuli $[F(1,23)=$ 13.46, $p<.001, \eta_{\mathrm{p}}^{2}=.37$ (nonsingleton vertical P3 $>$ nonsingleton horizontal P3)], as well as for 2-D stimuli $\left[F(1,23)=14.94, p<.001, \eta_{\mathrm{p}}^{2}=.39\right.$ (nonsingleton vertical P3 > nonsingleton horizontal P3)]. Response-locked P3 mean amplitudes also decreased as set size increased $\left[F(1,23)=9.44, p<.006, \eta_{\mathrm{p}}^{2}=.29\right.$; see Figure 6, lower right panel], without a statistically reliable modulation by type of stimuli, orientation conditions, or their interaction. In particular, the orientation $\times$ set size interaction failed to reach statistical significance $[F(1,23)=5.52, p=.03$, $\left.\eta_{\mathrm{p}}^{2}=.19\right]$.

Set size also affected stimulus-locked P3 peak latencies (four later than one), as evidenced by a significant set size main effect $\left[F(1,23)=13.66, p<.002, \eta_{\mathrm{p}}^{2}=.37\right]$. Neither type of gradient nor orientation nor any interaction involving these factors yielded statistically significant effects [all $F \mathrm{~s}(1,23) \leq 5.63, p \mathrm{~s} \geq .03, \eta_{p}^{2} \mathrm{~s} \leq .19$ ]. $\mathrm{P} 3$ peak latencies in the response-locked averages were influenced by set size $\left[F(1,23)=9.92, p<.005, \eta_{\mathrm{p}}^{2}=.30\right.$ (one earlier than four)], by the interaction between set size and target effects $\left[F(1,23)=8.46, p<.009, \eta_{\mathrm{p}}^{2}=.27\right]$, and by orientation conditions $\left[F(1,23)=17.96, p<.001, \eta_{\mathrm{p}}^{2}=.44\right.$ (vertical earlier than horizontal)].

\section{Discussion}

The present data are not consistent with the light-fromabove account for orientation anisotropy in VS tasks. On the one hand, we replicated behavioral (Experiment 1; Adams, 2007; Kleffner \& Ramachandran, 1992; Sun \& Perona, 1998; Thornton \& Gilden, 2007; Wolfe et al., 1999) as well as cortical (Experiment 1) orientation anisotropy under shape-from-shading conditions. On the other hand, neither behavioral nor cortical orientation anisotropy occurred exclusively under shape-from-shading conditions. In contrast, behavioral and cortical anisotropy occurred under bipartitioned conditions, in a manner that was completely indistinguishable from the shape-from-shading conditions.

Behavioral orientation anisotropy is therefore a very robust (see also Adams, 2007; Kleffner \& Ramachandran, 1992; Sun \& Perona, 1998; Thornton \& Gilden, 2007; Wolfe et al., 1999), yet nonspecific (see also van Zoest et al., 2006), phenomenon of VS. Furthermore, anisotropic cortical processing of vertical and horizontal visual stimuli seems to be a robust phenomenon as well, since both amplitude changes in the P1 and P3 latency range that were reported from Experiment 1 could be replicated in Experiment 2. Again, we found evidence of cortical anisotropy at multiple levels of the cortical hierarchy, and these anisotropies pointed in opposite directions. Specifically, whereas amplitudes in the P1 latency range were slightly more positive under horizontal orientations, P3 amplitudes were more positive under nonsingleton vertical orientations. Both ERP amplitude effects occurred in equal measure under shape-from-shading conditions, as well as under bipartitioned conditions.

The nonspecificity of behavioral and cortical orientation anisotropy is inconsistent with the light-from-above account for orientation anisotropy in VS tasks. According to this model of orientation anisotropy, to interpret visual stimuli three-dimensionally presupposes vertical-shading gradients such that the bias for overhead lighting is able to support perception of concavity or convexity, respectively. A 3-D interpretation of stimuli is not expected to occur in any of the remaining conditions of our VS task (Aks \& Enns, 1992). Therefore, behavioral and cortical anisotropy that we observed under bipartitioned conditions are clearly inconsistent with the light-from-above account for orientation anisotropy in VS tasks.

The item similarity model of orientation anisotropy (van Zoest et al., 2006) can explain the nonspecificity of behavioral anisotropy, yet the model does not provide a rationale for understanding the observed cortical anisotropies. Furthermore, its essential conjecture-namely, that 
targets and distractors are perceived as being more similar on horizontal than on vertical displays - needs to be tested empirically.

An alternative account for the observed orientation anisotropy in VS tasks may originate from some of the emergent properties of cortical visual neurons (for reviews, see Angelucci \& Bressloff, 2006; Carandini et al., 2005; Seriès, Lorenceau, \& Frégnac, 2003). Orientation tuning that is, responding optimally to a certain orientation and less to others - is among the most prominent properties of neurons in the striate cortex (Hubel \& Wiesel, 1962). Orientation selectivity is also the preferred property examined in neurophysiological studies of cortical visual neurons when contextual influences on spiking responses of visual neurons are under scrutiny. Specifically, presenting two stimuli concurrently, one in the receptive field of visual neurons and another in the spatial surround of the receptive field, usually results in a massive suppression of the spiking response. This surround suppression is maximal under iso-oriented surround conditions (i.e., when the receptive field and surround stimuli are identically oriented), whereas surround suppression is negligible under orthooriented surround conditions (i.e., when the receptive field and surround stimuli are perpendicular to each other).

Our model of orientation anisotropy in VS conveys the mechanisms of surround suppression to mid-level (extrastriate) vision, as illustrated in Figure 8. Both panels of Figure 8 show a system of neighboring perceptive fields (Jung \& Spillmann, 1970; Spillmann, 1994), ${ }^{3}$ exemplified by two perceptive fields, that are selectively responsive either to vertical (left panel) or to horizontal (right panel) input signals from low-level vision.

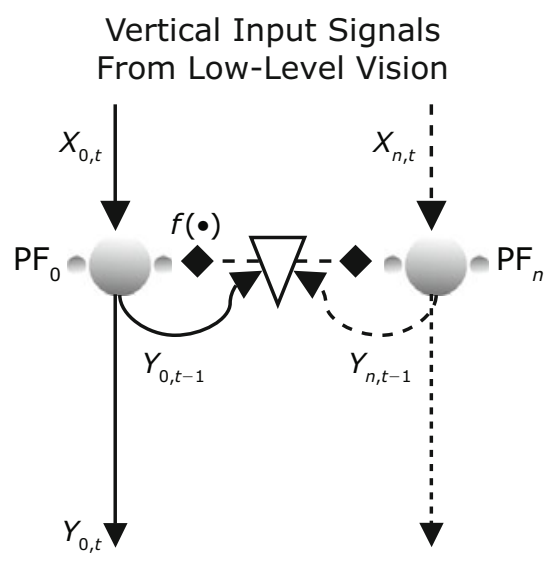

To High-Level Vision
Figure 8 describes how signal processing within a local network modulates signals in a particular perceptive field, here termed " $\mathrm{PF}_{0}$," at a particular point in discrete time, $t$. Specifically, $f(\cdot)$, the contextual filtering function, contributes to convert the input to $\mathrm{PF}_{0}, x_{0, t}\left(x_{0, t} \geq 0\right)$, to its output $y_{0, t}\left(y_{0, t} \geq 0\right)$. Two general assumptions about the architecture of this locally interconnected system of perceptive fields form the basis of our model of orientation anisotropy in VS. First, at time instant $t-1$, corollaries of output signals from each perceptive field are sent to cortical interneurons, exemplified by $y_{0, t-1}$ and $y_{n, t-1}$, with $y_{0, t-1} \geq 0$ and $y_{n, t-1} \geq 0$. Second, if two corollaries excite the interneurons concurrently, suppression at time instant $t$ is exhibited to the perceptive fields that have been the origin of the corollaries, exemplified by $f(\cdot) \geq 0$. Substantial suppression of the input signal to $\mathrm{PF}_{0}$ will result in case of simultaneous isomorphic input signals to the interneuron of comparable strength, thereby providing a mechanism for powerful contextual filtering suppressing locally homogeneous information represented in the perceptive fields. ${ }^{4}$

Our specific model comes next. We first suppose that the computational implementation of contextual filtering within this system of locally interconnected perceptive fields consists of divisive normalization (Albrecht \& Geisler, 1991; Heeger, 1992; Wainwright, Schwartz, \& Simoncelli, 2002). That is, if $y_{0, t}$ describes the output of $\mathrm{PF}_{0}$ at time $t$, it can be computed as

$$
y_{0, t}=\frac{x_{0, t}}{1+\beta \cdot \sum_{n=1}^{N} f\left(y_{0, t-1}, y_{n, t-1}\right)}, \quad n=1,2, \ldots, N,
$$

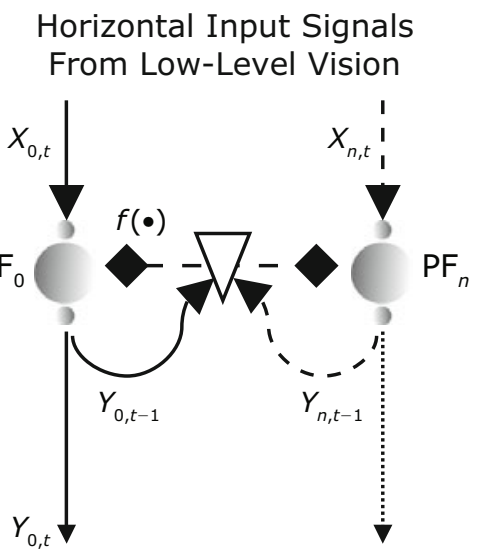

To High-Level Vision

Figure 8. A sketch of our model of contextual filtering in mid-level vision. Each panel shows a system of neighboring perceptive fields, exemplified by two perceptive fields $\left(\mathrm{PF}_{0}\right.$, $\mathrm{PF}_{n}$ ) that are selectively responsive either to vertical (left panel) or to horizontal (right panel) input signals from low-level vision. Note the anisotropy of the vertical and horizontal input signals from low-level vision $\left(x_{0, t}, x_{n, t}\right.$, vertical $<$ horizontal). The loops between perceptive fields give rise to contextual filtering by divisive normalization, leading to surround suppression in cases of isomorphic input signals to neighboring perceptive fields (i.e., when $x_{0, t}$ and $x_{n, t}$ excite $\mathrm{PF}_{0}$ and $\mathrm{PF}_{n}$ simultaneously). Multiplicative filtering functions are required to model an anisotropic response $\left(y_{0, t}\right.$, vertical $>$ horizontal). Triangles represent interneurons. Triangle-shaped arrowheads signify excitatory connections; diamond-shaped arrowheads represent inhibitory connections. Dashed lines indicate the conditionality of multiplicative contextual filtering. See the text for details. 
with $x_{0, t}$ representing the input signal at time $t, f\left(y_{0, t-1}\right.$, $\left.y_{n, t-1}\right) \geq 0$ being the inhibitory influence of $\mathrm{PF}_{n}$ on $\mathrm{PF}_{0}$ at time $t$, parameter $\beta \geq 0$ to be determined empirically, and $N$ denoting the number of connected perceptive fields that may impose a suppressive effect on $\mathrm{PF}_{0}$. Equation 1 can be interpreted as a contextual filtering function, parameterized via $f(\cdot)$ by corollaries of the time-lagged outputs, $y_{0, t-1}$ and $y_{n, t-1}$, respectively. A formal approach for deriving Equation 1 can be found in the Appendix.

Second, we suppose that the magnitude of the horizontal input from low-level vision surpasses the magnitude of the vertical input from low-level vision (see Figure 8). Our supposition that horizontal and vertical inputs are processed anisotropically at low-level vision can be defended on empirical grounds. B. Li, Peterson, and Freeman (2003) reported an orientation anisotropy with regard to the number of visual neurons that prefer certain orientations: There are more neurons (in the cat's striate cortex) with horizontal preference than there are neurons with preference for vertical or oblique orientations. The authors interpreted this orientation anisotropy as reflecting the fact that neural representations correlate with statistical properties of natural scenes (Geisler, 2008) at low-level vision in an adaptive manner (Simoncelli \& Olshausen, 2001). Thus, our observation of ERP amplitude anisotropies in the P1 latency range seems to reflect the anisotropic processing of vertical and horizontal content in low-level vision (vertical < horizontal; B. Li et al., 2003).

Third, we suggest a specific contextual filter function. Applying simple algebraic rules reveals that linear filter functions, $f(\cdot)=a * x$, are incapable of accounting for an anisotropic output of the system of perceptive fields that we specified above. The reason for this incapability of linear filter functions is that $x / \Sigma^{n} a * x$ is equivalent to $x / n * a * x$, which is equivalent to $1 / n * a$-that is, a quantity that is independent of $x$. Therefore, anisotropic input signals from low-level vision to perceptive fields (see above) will not be conserved in the output of these perceptive fields; their output will be constant when $x$ changes, because it will depend only on $n$ and $a$. To simulate an anisotropic output of the system of perceptive fields is possible if one considers quadratic filter functions, $f(\cdot)=x^{2}$. In this case, simplification of $x / \Sigma^{n} x^{2}$ yields $x / n * x^{2}$, which is equivalent to $1 / n * x$. Here, anisotropic input signals to perceptive fields will be conserved in the output of these perceptive fields, but in a reciprocal manner: The responses to larger input signals will be smaller, and vice versa (see Figure 8).

We therefore suggest a specific parameterization $f(\cdot)$ and, thereby, a specific contextual filtering function. The inhibitory loop between neighboring perceptive fields can be described as multiplicative:

$$
f\left(y_{0, t-1}, y_{n, t-1}\right)=y_{0, t-1} \cdot y_{n, t-1}, \quad n=1,2, \ldots, N \text {. }
$$

If $y_{0, t-1}=y_{n, t-1}=y$, we simply have $f\left(y_{0, t-1}\right.$, $\left.y_{n, t-1}\right)=y^{2}$. Interestingly, quadratic contextual filtering by divisive normalization has been advocated earlier in an attempt to account for nonlinear properties of visual neurons (Schwartz \& Simoncelli, 2001). On the other hand, if $y_{n, t-1}=0$, then $f\left(y_{0, t-1}, y_{n, t-1}\right)=0$, which means that
$\mathrm{PF}_{n}$ does not impose an inhibitory effect on $\mathrm{PF}_{0}$. Contextual filtering therefore seems to possess at least two major characteristics. First, the strength of the inhibitory effect on a perceptive field depends in a multiplicative manner on the outputs of the field itself and of each of its connected neighbors. In essence, then, this locally interconnected system of perceptive fields can be identified as a nonlinear system (Wu, David, \& Gallant, 2006). Second, in cases in which there are no output signals of neighboring perceptive fields, the inhibitory influence features conditionality, because its minimum is simply zero according to $f\left(y_{0, t-1}, y_{n, t-1}=0\right)=0$.

In a general sense, the mechanism of contextual filtering may provide crucial modulatory functions for visual processing. First, the primary function of contextual filtering is redundancy reduction (Barlow, 2001). Specifically, the saliency (Itti \& Koch, 2001) of large regions of isomorphic input signals will be greatly attenuated, whereas the saliency of nonisomorphic input signals, such as edges, boundaries, and contours, will be much less modified by contextual filtering. The accentuation of discontinuous regions of input signals may facilitate the segmentation of visual scenes and figure-ground separation. This analysis ultimately leads to a center-surround hypothesis for visual saliency (Gao, Mahadevan, \& Vasconcelos, 2008) that enables efficient coding at higher levels of visual analysis (Barlow, 2001). Second, the reciprocity of contextual filtering, as defined above, may be comparably important for visual processing: Salient low-level features will be integrated to nonsalient high-level bundles of features (and finally objects), and vice versa. The reciprocity of contextual filtering suggests that infrequently processed low-level visual features (originating from unpredictable, surprising, or even novel events and objects) gain a saliency advantage at higher levels of visual processing. This analysis ultimately leads to probabilistic models of visual saliency (Itti \& Baldi, 2009) if one considers that low-level visual processing reflects the statistical properties of natural scenes in an adaptive manner (Geisler, 2008; Simoncelli \& Olshausen, 2001).

The proposed contextual-filtering model explains the behavioral orientation anisotropy observed in our VS studies via its supposed modulatory influences on visual saliency. Specifically, the initial processing advantage of horizontal content at low levels of visual analysis is assumed to become reversed at those levels of visual processing that follow contextual filtering, thereby slowing VS for horizontal content. In this regard, it is worth mentioning that a distinct behavioral orientation anisotropy effect occurred exclusively in nonsingleton conditions of our VS tasks, as if its occurrence depends considerably on contextual filtering. Z. Li $(1999,2002)$ argued that many basic phenomena of VS (Wolfe, 2007) could be traced back to receptive field-surround interactions of visual cortical neurons (see also Nothdurft, 1991, for another contextual model of VS). The details and the persuasiveness of these two models of VS are beyond the scope of this article and will not be portrayed here.

The proposed contextual-filtering model also explains why the two observed cortical anisotropy effects pointed in opposite directions. We conjectured above that the 
anisotropic ERP amplitudes in the P1 latency range reflect vertical and horizontal content being processed anisotropically in low-level vision (vertical < horizontal; B. Li et al., 2003). In agreement with this claim, the horizontal enhancement of ERP amplitudes in the P1 latency range was observed independently of set size conditions and, thus, independently of the conditionality of contextual filtering. Furthermore, the anisotropic P1 and P3 amplitude changes pointed in opposite directions, because we measured a horizontal enhancement of ERP amplitudes in the P1 latency range but a vertical enhancement of P3 amplitudes. This pattern of ERP amplitude effects is compatible with the reciprocity of the contextual-filtering model if one assumes that whereas ERP amplitudes in the P1 latency range reflect input to the proposed system of neighboring perceptive fields, $\mathrm{P} 3$ amplitudes reflect their output. In line with this conjecture, recall that whereas the horizontal enhancement of ERP amplitudes in the P1 latency range occurred independently of set size conditions, we observed that the vertical enhancement of $\mathrm{P} 3$ amplitudes depended on set size conditions: It occurred exclusively in response to four-item displays, as if its occurrence is considerably dependent on the effects of contextual filtering. Finally, the mere existence of a set size main effect on P3, but not P1, amplitudes (P3 set size four $<$ P3 set size one; see also Kok, 2001) is compatible with assuming that $P 3$, but not $\mathrm{P} 1$, amplitudes are massively influenced by contextual filtering. Overall, the proposed model is remarkably successful in accounting not only for behavioral orientation anisotropy, but also for the fact that the two observed cortical anisotropy effects pointed in opposite directions and that they were differentially affected by set size.

The context model accounted for the behavioral and cortical anisotropies that we observed in our own experiments. It can also account for the body of work on anisotropies in shape-from-shading VS studies (Adams, 2007; Kleffner \& Ramachandran, 1992; Sun \& Perona, 1998; Thornton \& Gilden, 2007; Wolfe et al., 1999) and in van Zoest et al.'s (2006) study (Experiments 1-3). Its explanatory power is limited only by the data from van Zoest et al.'s Experiment 4, in which no orientation anisotropy was found under conditions of interitem asymmetry. However, these findings might be compatible with our context model if perceptive fields do not exhibit only orientation specificity, but are also specific with regard to other (yet to be determined) features of the visual input. Given this to be true, the context model of anisotropy predicts less pronounced anisotropy when dissimilar, as compared with when similar, stimuli occur in the search displays. For example, if perceptive fields are tuned for a combination of orientation and spatial frequency, the context model predicts stronger orientation anisotropy when stimuli have the same spatial frequency, as compared with when spatial frequency differs between stimuli.

We found only one study that directly examined the effects of manipulating target-distractor orientation (Wolfe et al., 1999, Experiments 1 and 4). Here, search for targets rotated $90^{\circ}$ from the distractors was more efficient than search for targets rotated $180^{\circ}$ from the distractors. These findings are clearly compatible with predictions of our context model. Additional evidence that visual information processing is strongly modulated by contextual interaction comes from psychophysical studies in which observers had to discriminate the orientation of lines. When tested with simple stimuli, these studies showed that performance is best for horizontal and vertical orientations and worst for oblique orientations (the oblique effect; Essock, DeFord, Hansen, \& Sinai, 2003; Hansen \& Essock, 2004). However, these authors showed that, when tested with more complex images consisting of naturalistic content, performance is best for oblique and vertical orientations and worst for horizontal orientations (the horizontal effect $)$. They interpreted this horizontal effect as being the consequence of minimizing the visual saliency of the horizontal content under more naturalistic conditions.

The context model of VS is similar to earlier models of VS (Z. Li, 1999, 2002; Nothdurft, 1991), and it is consistent with some of the most important VS phenomena (see Wolfe, 2007, for a review of these phenomena), such as the set size effect (by increasing $n$ in Equation 1), the target absence effect (target-absent displays are necessarily more homogeneous than target-present displays), and the targetdistractor similarity effect (by increasing the sum over the $y \cdot y$ products in the denominator of Equation 1 after insertion of Equation 2). It rests on a simple mechanism of lateral inhibition that is supposed to occur ubiquitously in the whole brain and that has been studied most elegantly in the visual system (for reviews, see Angelucci \& Bressloff, 2006; Carandini et al., 2005; Seriès et al., 2003).

We proposed here a simple, yet highly integrative, model of behavioral and electrophysiological properties of orientation anisotropy in VS. Our context model highlights local suppressive interactions as a cortical mechanism of information processing, putatively achieved through lateral inhibition. The pursuance of appropriate research on orientation anisotropy may well help to decide between two fundamentally contrasting views of visual perception. The light-from-above model of orientation anisotropy is explicitly a model of indirect perception (Kersten et al., 2004; Ramachandran, 1988; Ramachandran \& RogersRamachandran, 2008). In these models, visual perception is thought to involve the activation of nonsensory knowledge, such as beliefs, memories, and inferences. According to direct models of visual perception, optical stimulation is extraordinarily rich and provides such a precise specification of the environment that perceivers need only detect the appropriate information without nonsensory contributions (see Michaels \& Carello, 1981, for a comprehensive review of the distinctions between indirect and direct models of visual perception). The context model of orientation anisotropy is consistent with the direct view of visual perception, since it attributes anisotropy to lateral processes within the visual system itself.

\section{AUTHOR NOTE}

J.K. is now at the Department of Psychology, Philipps University Marburg. Thanks are due David Gilden, University of Texas at Austin, for providing his stimulus materials. Steve Luck, University of California, Davis, and three anonymous reviewers offered many valuable comments and suggestions for improving earlier drafts of the manuscript. We also thank Wolfgang Skrandies, Justus Liebig University, Giessen, and 
Tim Fingscheidt, University of Technology Carolo-Wilhelmina, Braunschweig, for numerous suggestions. Their ideas were very valuable for improving the content of this article. Correspondence concerning this article should be addressed to B. Kopp, Cognitive Neurology, University of Technology Carolo-Wilhelmina Braunschweig, Braunschweig, Germany (e-mail: b.kopp@klinikum-braunschweig.de).

\section{REFERENCES}

Adams, W. J. (2007). A common light-prior for visual search, shape and reflectance judgments. Journal of Vision, 7(11, Art. 11), 1-7.

Adams, W. J., Graf, E. W., \& ERNST, M. O. (2004). Experience can change the "light-from-above" prior. Nature Neuroscience, 7, 10571058 .

AKs, D. J., \& EnNs, J. T. (1992). Visual search for direction of shading is influenced by apparent depth. Perception \& Psychophysics, 52, 63-74

Albrecht, D. G., \& Geisler, W. S. (1991). Motion selectivity and the contrast-response function of simple cells in the visual cortex. Visual Neuroscience, 7, 531-546.

ANGelucCI, A., \& Bressloff, P. C. (2006). Contribution of feedforward, lateral and feedback connections to the classical receptive field center and extra-classical receptive field surround of primate V1 neurons. Progress in Brain Research, 154, 93-120.

Barenholtz, E., Cohen, E. H., Feldman, J., \& Singh, M. (2003). Detection of change in shape: An advantage for concavities. Cognition, 89, 1-9.

BARLOw, H. (2001). Redundancy reduction revisited. Network: Computation in Neural Systems, 12, 241-253.

BREWSTER, D. (1826). On the optical illusion of the conversion of cameos into intaglios and of intaglios into cameos with an account of other analogous phenomena. Edinburgh Journal of Science, 4, 99-108.

Carandini, M., Demb, J. B., Mante, V., Tolhurst, D. J., Dan, Y., Olshausen, B. A., ET AL. (2005). Do we know what the early visual system does? Journal of Neuroscience, 25, 10577-10597.

Chun, M. M., \& Wolfe, J. M. (1996). Just say no: How are visual searches terminated when there is no target present? Cognitive Psychology, 30, 39-78.

Di Russo, F., Aprile, T., Spitoni, G., \& SPinelli, D. (2007). Impaired visual processing of contralesional stimuli in neglect patients: A visual-evoked potential study. Brain, 131, 842-854.

Di Russo, F., Martínez, A., Sereno, M. I., Pitzalis, S., \& Hillyard, S. A. (2002). Cortical sources of the early components of the visual evoked potential. Human Brain Mapping, 15, 95-111.

Duncan, J., \& Humphreys, G. W. (1989). Visual search and stimulus similarity. Psychological Review, 96, 433-458.

Essock, E. A., DeFord, J. K., Hansen, B. C., \& Sinai, M. J. (2003). Oblique stimuli are seen best (not worst!) in naturalistic broad-band stimuli: A horizontal effect. Vision Research, 43, 1329-1335.

Gao, D., Mahadevan, V., \& Vasconcelos, N. (2008). On the plausibility of the discriminant center-surround hypothesis for visual saliency. Journal of Vision, 8(7, Art. 13), 1-18.

GEISLER, W. S. (2008). Visual perception and the statistical properties of natural scenes. Annual Review of Psychology, 59, 167-192.

Gratton, G., Coles, M. G., \& Donchin, E. (1983). A new method for off-line removal of ocular artifact. Electroencephalography \& Clinical Neurophysiology, 55, 468-484.

HANSEN, B. C., \& Essock, E. A. (2004). A horizontal bias in human visual processing of orientation and its correspondence to the structural components of natural scenes. Journal of Vision, 4, 1044-1060.

HEEGER, D. J. (1992). Normalization of cell responses in cat striate cortex. Visual Neuroscience, 9, 181-197.

Hillyard, S. A., Teder-SälejÄrvi, W. A., \& Münte, T. F. (1998). Temporal dynamics of early perceptual processing. Current Opinion in Neurobiology, 8, 202-210.

Hou, C., Pettet, M. W., Vilddavski, V. Y., \& Norcia, A. M. (2006). Neural correlates of shape-from-shading. Vision Research, 46, 10801090.

Hubel, D. H., \& Wiesel, T. N. (1962). Receptive fields, binocular interaction and functional architecture in the cat's visual cortex. Journal of Physiology, 160, 106-154.

ITTI, L., \& BALDI, P. (2009). Bayesian surprise attracts human attention. Vision Research, 49, 1295-1306.
ITTI, L., \& Косн, C. (2001). Computational modelling of visual attention. Nature Reviews Neuroscience, 2, 194-203.

Jung, R., \& Spillmann, L. (1970). Receptive-field estimation and perceptual integration in human vision. In F. A. Young \& D. B. Lindsley (Eds.), Early experience and visual information processing in perceptual and reading disorders (pp. 181-197). Washington, DC: National Academy of Sciences.

Kersten, D., Mamassian, P., \& Yuille, A. (2004). Object perception as Bayesian inference. Annual Review of Psychology, 55, 271-304.

KLefFner, D. A., \& Ramachandran, V. S. (1992). On the perception of shape from shading. Perception \& Psychophysics, 52, 18-36.

KoK, A. (2001). On the utility of P3 amplitude as a measure of processing capacity. Psychophysiology, 38, 557-577.

KopP, B. (2008). The P300 component of the event-related brain potential and Bayes' theorem. In M.-K. Sun (Ed.), Cognitive sciences at the leading edge (pp. 87-96). New York: Nova.

LeE, T. S., Yang, C. F., Romero, R., \& Mumford, D. (2002). Neural activity in early visual cortex reflects behavioral experience and higherorder perceptual saliency. Nature Neuroscience, 5, 589-597.

Li, B., Peterson, M. R., \& Freeman, R. D. (2003). Oblique effect: A neural basis in the visual cortex. Journal of Neurophysiology, $\mathbf{9 0}$, 204-217.

LI, Z. (1999). Contextual influences in V1 as a basis for pop out and asymmetry in visual search. Proceedings of the National Academy of Sciences, 96, 10530-10535.

LI, Z. (2002). A saliency map in primary visual cortex. Trends in Cognitive Sciences, 6, 9-16.

LuCK, S. J. (2005). An introduction to the event-related potential technique. Cambridge, MA: MIT Press.

LUCK, S. J., \& HiLlYARD, S. A. (1990). Electrophysiological evidence for parallel and serial processing during visual search. Perception \& Psychophysics, 48, 603-617.

Mamassian, P., Jentzsch, I., Bacon, B. A., \& Schweinberger, S. R. (2003). Neural correlates of shape from shading. NeuroReport, 14, 971-975.

Michaels, C. F., \& Carello, C. (1981). Direct perception. Englewood Cliffs, NJ: Prentice Hall.

MilLeR, J. (1988). A warning about median reaction time. Journal of Experimental Psychology: Human Perception \& Performance, 14, 539-543.

NothduRFt, H. C. (1991). Texture segmentation and pop-out from orientation contrast. Vision Research, 31, 1073-1078.

PALMER, J. (1995). Attention in visual search: Distinguishing four causes of a set size effect. Current Directions in Psychological Science, 4, 118-123.

Picton, T. W., Bentin, S., Berg, P., Donchin, E., Hillyard, S. A., JohnSON, R., JR., ET AL. (2000). Guidelines for using human eventrelated potentials to study cognition: Recording standards and publication criteria. Psychophysiology, 37, 127-152.

Prados, E., \& Faugeras, O. (2006). Shape from shading. In N. Paragios, Y. Chen, \& O. Faugeras (Eds.), Handbook of mathematical models in computer vision (pp. 375-388). Berlin: Springer.

RamachandRan, V. S. (1988). Perception of shape from shading. Nature, 331, 163-166.

Ramachandran, V. S., \& Rogers-Ramachandran, D. (2008). Seeing is believing. Scientific American Mind, 19, 16-18.

Schwartz, O., \& Simoncelli, E. P. (2001). Natural signal statistics and sensory gain control. Nature Neuroscience, 4, 819-825.

Seriès, P., Lorenceau, J., \& Frégnac, Y. (2003). The "silent" surround of V1 receptive fields: Theory and experiments. Journal of Physiology, 97, 453-474.

Simoncelli, E. P., \& Olshausen, B. A. (2001). Natural image statistics and neural representation. Annual Review of Neuroscience, 24, 1193-1216.

Spencer, K. M., Dien, J., \& Donchin, E. (2001). Spatiotemporal analysis of the late ERP responses to deviant stimuli. Psychophysiology, 38, 343-358.

SpillmanN, L. (1994). The Hermann grid illusion: A tool for studying human perceptive field organization. Perception, 23, 691-708.

Sun, J., \& Perona, P. (1998). Where is the sun? Nature Neuroscience, 1, 183-184.

Thornton, T. L., \& Gilden, D. L. (2007). Parallel and serial processes in visual search. Psychological Review, 114, 71-103. 
Treisman, A. M., \& Gelade, G. (1980). A feature-integration theory of attention. Cognitive Psychology, 12, 97-136.

Treisman, A. M., \& Gormican, S. (1988). Feature analysis in early vision: Evidence from search asymmetries. Psychological Review, 95, $15-48$.

van Zoest, W., Giesbrecht, B., Enns, J., \& Kingstone, A. (2006). New reflections in visual search: Interitem symmetry matters! Psychological Science, 17, 535-542.

Wainwright, M. J., Schwartz, O., \& Simoncelli, E. P. (2002). Natural image statistics and divisive normalization: Modeling nonlinearities and adaptation in cortical neurons. In R. P. N. Rao, B. A. Olshausen, \& M. S. Lewicki (Eds.), Probabilistic models of the brain: Perception and neural function (pp. 203-222). Cambridge, MA: MIT Press.

Wolfe, J. M. (1998). What can one million trials tell us about visual search? Psychological Science, 9, 33-39.

Wolfe, J. M. (2007). Guided Search 4.0: Current progress with a model of visual search. In W. Gray (Ed.), Integrated models of cognitive systems (pp. 99-119). New York: Oxford University Press.

Wolfe, J. M., \& Horowitz, T. S. (2004). What attributes guide the deployment of visual attention and how do they do it? Nature Reviews Neuroscience, 5, 495-501.

Wolfe, J. M., Klempen, N. L., \& Shulman, E. P. (1999). Which end is up? Two representations of orientation in visual search. Vision Research, 39, 2075-2086.

Wolfe, J. M., Yee, A., \& Friedman-Hill, S. R. (1992). Curvature is a basic feature for visual search tasks. Perception, 21, 465-480.

Wu, M. C., David, S. V., \& Gallant, J. L. (2006). Complete functional characterization of sensory neurons by system identification. Annual Review of Neuroscience, 29, 477-505.

\section{NOTES}

1. Medians are used in the RT calculations, which has the advantage of minimizing the effects of extreme data points. However, Miller (1988) has pointed out that the use of medians introduces a bias that depends on the number of values going into the median. Thus, medians can potentially be problematic in experiments, such as Experiment 1, in which there are large differences in the numbers of trials in different conditions, because different conditions will show different amounts of bias. Therefore, it is important to note that we used equal numbers of trials across conditions in Experiment 2. The replication of all essential RT findings across both experiments argues against a sizable role of a bias depending on the numbers of values going into the median.

2. We focus our arguments on the change in voltage in the P1 latency range in the following (rather than on those in the N1 latency range) mainly for two reasons. First, the P1 occurs earlier than the N1. Second, amplitude changes in the P1 latency range were much more consistent than amplitude changes in the N1 latency range across our two experiments. Specifically, the modulation of amplitudes in the $\mathrm{P} 1$ latency range by shading orientation was statistically more clear-cut across our two experiments than the modulation of amplitudes in the N1 latency range by the main effect of this factor.

3. Perceptive fields are analogous to receptive fields, but at the psychophysical level (Jung \& Spillmann, 1970). Much research on perceptive fields has been conducted on the Hermann grid illusion (Spillmann, 1994). Receptive fields and perceptive fields may share orientation tuning as one of the principles of selective responsiveness. However, whereas receptive fields are tuned toward the detection of lines in a specific orientation, perceptive fields may be tuned toward more complex arrangements of the orientation of light. For example, the verticality of the step difference of vertical bipartitioned stimuli contrasts with the horizontal edge that these stimuli contain. Apparently, the orientation of the shading gradient or of the step difference is the feature that propels the selective responsiveness of perceptive fields (see also the concept of an orientation axis in Wolfe et al., 1999).

4. Iso-oriented stimuli seem to be isomorphic, irrespective of their polarity (see also Wolfe et al., 1999). For example, verticality may suffice for isomorphism, no matter whether neighboring stimuli are white above or white below.

\section{APPENDIX}

In its most general form, the model can be written as

$$
y_{0, t}=\frac{A \cdot x_{0, t}}{B+C \cdot \sum_{n=1}^{N} f\left(y_{0, t-1}, y_{n, t-1}\right)}, \quad n=1,2, \ldots, N .
$$

Without loss of generality, the number of free parameters can be reduced as follows:

$$
y_{0, t}=\frac{x_{0, t}}{\frac{B}{A}+\frac{C}{A} \cdot \sum_{n=1}^{N} f\left(y_{0, t-1}, y_{n, t-1}\right)}, \quad n=1,2, \ldots, N .
$$

In the complete absence of contextual filtering - that is, when all $f\left(y_{0, t-1}, y_{n, t-1}\right)=0$-Equation A2 is then transparent- that is, $y_{0, t}=x_{0, t}$, if $B / A=1$. This analysis leads to our specific formulation of the contextual filtering function,

$$
y_{0, t}=\frac{x_{0, t}}{1+\beta \cdot \sum_{n=1}^{N} f\left(y_{0, t-1}, y_{n, t-1}\right)}, \quad n=1,2, \ldots, N
$$

with $\beta=C / A$. Equation A3 equals Equation 1.

This approach to deriving the contextual filtering function was brought to our attention by Tim Fingscheidt, who argued, and we agree, that the function needs a more comprehensive formulation. We honestly thank him for his valuable suggestion. 